\title{
Decision-Making Framework for an Effective Sanitizer to Reduce COVID-19 under Fermatean Fuzzy Environment
}

\author{
Muhammad Akram $\left(\mathbb{D},{ }^{1}\right.$ Gulfam Shahzadi, ${ }^{1}$ and Abdullah Ali H. Ahmadini ${ }^{2}$ \\ ${ }^{1}$ Department of Mathematics, University of the Punjab, New Campus, Lahore, Pakistan \\ ${ }^{2}$ Department of Mathematics, Faculty of Science, Jazan University, Jazan, Saudi Arabia \\ Correspondence should be addressed to Muhammad Akram; m.akram@pucit.edu.pk
}

Received 13 July 2020; Accepted 29 August 2020; Published 30 October 2020

Academic Editor: Tahir Mahmood

Copyright (c) 2020 Muhammad Akram et al. This is an open access article distributed under the Creative Commons Attribution License, which permits unrestricted use, distribution, and reproduction in any medium, provided the original work is properly cited.

\begin{abstract}
The purpose of this article is to develop some general aggregation operators (AOs) based on Einstein's norm operations, to cumulate the Fermatean fuzzy data in decision-making environments. A Fermatean fuzzy set (FFS), possessing the more flexible structure than the intuitionistic fuzzy set (IFS) and Pythagorean fuzzy set (PFS), is a competent tool to handle vague information in the decision-making process by the means of membership degree (MD) and nonmembership degree (NMD). Our target is to empower the AOs using the theoretical basis of Einstein norms for the FFS to establish some advantageous operators, namely, Fermatean fuzzy Einstein weighted averaging (FFEWA), Fermatean fuzzy Einstein ordered weighted averaging (FFEOWA), generalized Fermatean fuzzy Einstein weighted averaging (GFFEWA), and generalized Fermatean fuzzy Einstein ordered weighted averaging (GFFEOWA) operators. Some properties and important results of the proposed operators are highlighted. As an addition to the MADM strategies, an approach, based on the proposed operators, is presented to deal with Fermatean fuzzy data in MADM problems. Moreover, multiattribute decision-making (MADM) problem for the selection of an effective sanitizer to reduce coronavirus is presented to show the capability and proficiency of this new idea. The results are compared with the Fermatean fuzzy TOPSIS method to exhibit the potency of the proposed model.
\end{abstract}

\section{Introduction}

In decision sciences, it is an important aspect to find the ranking order of the alternatives corresponding to different attributes according to the preferences of the decisionmaking experts. Therefore, selection of various attributes of the alternatives is a very complex task. These decisions cannot be interpreted by the exact data so the need of a powerful model was raised to handle the ambiguous data. For that issue, Zadeh [1] initiated the innovative idea of fuzzy set (FS) which served as the backbone of the FS theory. FS permits the experts to describe their satisfaction level (membership degree) regarding performance of a member within the unit interval. Although, the FSs provide the grounds to the uncertain assessments but they were not adequate enough to describe the NMD. To overcome the limitations of FS, Atanassov [2] introduced a more dominant model, namely, IFS which has both MD $\mu$ and NMD $v$ with condition $\mu+v \leq 1$. The theory of IFS was felt to be inept and insufficient to represent the inexact data as there are a lot of problems where the sum of MD and NMD is exceeded by 1 . To reduce such type of complications, Yager [3] delivered the idea of PFS with condition $\mu^{2}+v^{2} \leq 1$. However, PFS has also some limitations if MD of an element is 0.8 and NMD is 0.7 , then sum of square of these values is greater than 1 . Then, Yager [4] developed the theory of $q$-rung orthopair fuzzy set ( $q$-ROFS) with condition $\mu^{q}+v^{q} \leq 1$. Recently, Senapati and Yager [5] gave the concept of FFS as a generalization of IFS and PFS.

The worthwhile theory of AOs is widely applied to decisionmaking scenarios for the sake of data aggregation and to identify the best alternative from the possible choices. $\mathrm{Xu}$ [6] gave the idea of intuitionistic fuzzy (IF) AOs. The concept of generalized AOs for IFS was developed by Zhao et al. [7]. Rahman et al. [8] 
introduced Pythagorean fuzzy (PF) AOs. Zhao and Wei [9] studied the Einstein hybrid AOs under IF environment. The idea of IF aggregation using Einstein operations was discussed by Wang and Liu [10]. The induced interval-valued IF Einstein AOs were developed by Cai and Han [11]. Garg [12] studied the generalized PF Einstein weighted arithmetic AOs. Garg [13] also proposed the generalized PF Einstein weighted geometric AOs. The Pythagorean Dombi fuzzy AOs with applications were discussed by Akram et al. [14]. Shahzadi et al. [15] proposed the decision-making approach using PF Yager AOs. Liu and Wang [16] expressed $q$-rung orthopair fuzzy $(q-\mathrm{ROF})$ weighted AOs. Wei et al. [17] studied weighted Heronian mean AOs under $q$-ROF information. $q$-ROF power Maclaurin AOs were developed by Liu et al. [18]. Jana et al. [19] studied Dombi AOs for $q$-ROFS. Liu and Liu [20] proposed $q$-ROF Bonferroni mean operators. Joshi and Gegov [21] studied the confidence levels $q$-ROF aggregation operators. Akram and Shahzadi [22] developed the hybrid decision-making model under $q$-ROF Yager AOs. Liu et al. [23] extended the concept of prioritized weighted AOs for complex $q$-ROFS. Senapati and Yager [24] studied subtraction, division, and Fermatean arithmetic mean operations over FFS. The idea of Fermatean fuzzy (FF) weighted averaging/geometric operators was also given by Senapati and Yager [25]. For more information and applications, the readers can refer to [26-59].

The motivations of this article are described as follows:

(1) The judgement of a perfect alternative in an FF environment is a laborious MADM problem. The prevalent model, possessing the more space than the IF model and PF model, vigorously elaborates the imprecise decisions for the selection of best alternative.

(2) As Einstein AOs are the simplest and quite creative approach for dealing with DM affairs, basically, this article directs Einstein AOs in FF surroundings to face complex issues.

(3) The outcomes based on conclusion are quite accurate under Einstein AOs when it is put on to the realitybased MADM problems in FF data.

(4) The proposed operators are keen to provide the optimal solution not only for FF environment but also to work efficiently for IF and PF environment.

The contributions of this article are described as follows:

(1) The feasibility of FFNs is merged with the aggregation skills of Einstein norms to establish more powerful, multiskilled, and practical AOs which can be deployed to aggregate FF data and to get more accuracy in decision-making scenarios

(2) The dominant properties as well as the notable results of the proposed operators are highlighted

(3) An algorithm is studied to handle complex realistic problems with FF data

(4) A MADM problem for the selection of an effective sanitizer to reduce coronavirus is discussed by using proposed operators
(5) A validity test is discussed for the approval and authenticity of proposed theory

(6) At the end, the benefits and characteristics of the proposed work are discussed by comparison analysis

The remaining paper is as follows. In Section 2, we recall the concept of FFS and related score functions. Section 3 provides Einstein operational laws for FFNs. In Sections 4 and 5, we study the FFEWA and FFEOWA operators, respectively, and related properties to them. In Sections 6 and 7, we present the idea of GFFEWA and GFFEOWA operators, respectively. In Section 8, we propose an algorithm for our new model and discuss a MADM problem for the selection of a good sanitizer to reduce the coronavirus. Section 9 provides the validity criteria to prove the consistency of the proposed work. Section 10 gives the comparison analysis of proposed theory with the FF TOPSIS method. In Section 11, we have concluded the results related to the proposed model.

The list of acronyms in research paper is given in Table 1.

\section{Preliminaries}

In this section, we recall some basic definitions including IFS, PFS, FFS, and score functions related to FFS.

Definition 1. (see [2]). An IFS I on nonempty set $\mathscr{V}$ is given by

$$
I=\left\{\left\langle x, \mu_{I}(x), v_{I}(x)\right\rangle\right\},
$$

where $\mu_{I}: \mathscr{V} \longrightarrow[0,1]$ and $\nu_{I}: \mathscr{V} \longrightarrow[0,1]$ specify MD and NMD of an element $x \in \mathscr{V}$, respectively. $\varpi_{I}(x)=1-$ $\mu_{I}(x)-v_{I}(x)$ is indeterminacy degree $(\mathrm{InD})$ of an element $x \in \mathscr{V}$.

Definition 2. (see [3]). A PFS $P$ on nonempty set $\mathscr{V}$ is given by

$$
P=\left\{\left\langle x, \mu_{P}(x), v_{P}(x)\right\rangle\right\},
$$

where $\mu_{P}: \mathscr{V} \longrightarrow[0,1]$ and $v_{P}: \mathscr{V} \longrightarrow[0,1]$ specify $\mathrm{MD}$ and NMD of an element, respectively. $\omega_{P}(x)=$ $\sqrt{1-\left(\mu_{P}(x)\right)^{2}-\left(\nu_{P}(x)\right)^{2}}$ is $\operatorname{InD}$.

Definition 3. (see [5]). An FFS $\mathscr{R}$ on nonempty set $\mathscr{V}$ is given by

$$
\mathscr{R}=\left\{\left\langle x, \mu_{\mathscr{R}}(x), v_{\mathscr{R}}(x)\right\rangle\right\},
$$

where $\mu_{\mathscr{R}}: \mathscr{V} \longrightarrow[0,1], v_{\mathscr{R}}: \mathscr{V} \longrightarrow[0,1]$, and ${\varpi_{\mathscr{R}}}(x)=$ $\sqrt[3]{1-\left(\mu_{\mathscr{R}}(x)\right)^{3}-\left(\nu_{\mathscr{R}}(x)\right)^{3}}$ specify MD, NMD, and $\mathrm{InD}$, respectively. FFNs are components of the FFS.

Definition 4. (see [5]). The score function and accuracy function for FFN $\mathscr{R}=\left(\mu_{\mathscr{R}}, v_{\mathscr{R}}\right)$ are represented by

$$
\begin{array}{ll}
S(\mathscr{R})=\mu_{\mathscr{R}}^{3}-v_{\mathscr{R}}^{3}, & \text { where } S(\mathscr{R}) \in[-1,1], \\
\mathscr{A}(\mathscr{R})=\mu_{\mathscr{R}}^{3}+v_{\mathscr{R}}^{3}, & \text { where } \mathscr{A}(\mathscr{R}) \in[0,1] .
\end{array}
$$


TABLE 1: List of acronyms.

\begin{tabular}{lc}
\hline Acronyms & Description \\
\hline FS & Fuzzy set \\
IFS & Intuitionistic fuzzy set \\
PFS & Pythagorean fuzzy set \\
$q$-ROFS & $q$-rung orthopair fuzzy set \\
FFS & Fermatean fuzzy set \\
FFN & Fermatean fuzzy number \\
AOs & Aggregation operators \\
MADM & Multiattribute decision-making \\
FF TOPSIS & Fermatean fuzzy TOPSIS \\
\hline
\end{tabular}

Definition 5. (see [5]). Consider two FFNs $\mathscr{R}_{1}=\left\langle\mu_{\mathscr{R}_{1}}, \nu_{\mathscr{R}_{1}}\right\rangle$ and $\mathscr{R}_{2}=\left\langle\mu_{\mathscr{R}_{2}}, \nu_{\mathscr{R}_{2}}\right\rangle$. Then,

(1) If $S\left(\mathscr{R}_{1}\right)<S\left(\mathscr{R}_{2}\right)$, then $\mathscr{R}_{1} \prec \mathscr{R}_{2}$.

(2) If $S\left(\mathscr{R}_{1}\right)>S\left(\mathscr{R}_{2}\right)$, then $\mathscr{R}_{1}>\mathscr{R}_{2}$.

(3) If $S\left(\mathscr{R}_{1}\right)=S\left(\mathscr{R}_{2}\right)$, then
(a) If $\mathscr{A}\left(\mathscr{R}_{1}\right)<\mathscr{A}\left(\mathscr{R}_{2}\right)$, then $\mathscr{R}_{1} \prec \mathscr{R}_{2}$.
(b) If $\mathscr{A}\left(\mathscr{R}_{1}\right)>\mathscr{A}\left(\mathscr{R}_{2}\right)$, then $\mathscr{R}_{1}>\mathscr{R}_{2}$.
(c) If $\mathscr{A}\left(\mathscr{R}_{1}\right)=\mathscr{A}\left(\mathscr{R}_{2}\right)$, then $\mathscr{R}_{1} \sim \mathscr{R}_{2}$.

\section{Einstein Operational Law of FFNs}

In this section, we present concepts of the Einstein $t$-norm and $t$-conorm operations for FFNs and some of their properties. The Einstein operations on FFNs are defined as follows.

Definition 6. Let $\mathscr{R}=\langle\mu, v\rangle, \mathscr{R}_{1}=\left\langle\mu_{1}, v_{1}\right\rangle$, and $\mathscr{R}_{2}=$ $\left\langle\mu_{2}, \nu_{2}\right\rangle$ be FFNs and $\lambda>0$; then,

(i) $\overline{\mathscr{R}}=\left\langle v_{\mathscr{R}}, \mu_{\mathscr{R}}\right\rangle$

(ii) $\mathscr{R}_{1} \wedge_{\varepsilon} \mathscr{R}_{2}=\left\langle\min \left\{\mu_{1}, \mu_{2}\right\}, \max \left\{v_{1}, v_{2}\right\}\right\rangle$

(iii) $\mathscr{R}_{1} \vee_{\varepsilon} \mathscr{R}_{2}=\left\langle\max \left\{\mu_{1}, \mu_{2}\right\}, \min \left\{\nu_{1}, v_{2}\right\}\right\rangle$

(iv) $\mathscr{R}_{1} \oplus_{\varepsilon} \mathscr{R}_{2}=\left\langle\sqrt[3]{\left(\mu_{1}^{3}+\mu_{2}^{3}\right) /\left(1+\mu_{1}^{3} \cdot{ }_{\varepsilon} \mu_{2}^{3}\right)},\left(\nu_{1} \cdot{ }_{\varepsilon} \nu_{2} /\right.\right.$ $\sqrt[3]{\left.\left.1+\left(1-v_{1}^{3}\right) \cdot \varepsilon\left(1-v_{2}^{3}\right)\right)\right\rangle}$

(v) $\mathscr{R}_{1} \oplus_{\varepsilon} \mathscr{R}_{2}=\left\langle\left(\mu_{1} \cdot{ }_{\varepsilon} \mu_{2} / \sqrt[3]{1+\left(1-\mu_{1}^{3}\right) \cdot \varepsilon\left(1-\mu_{2}^{3}\right)}\right)\right.$, $\sqrt[3]{\left.\left(\nu_{1}^{3}+v_{2}^{3}\right) /\left(1+\nu_{1}^{3} \cdot \varepsilon_{2}^{3}\right)\right\rangle}$

(vi) $\lambda \cdot{ }_{\varepsilon} \mathscr{R}=\left\langle\sqrt[3]{\left(\left(1+\mu^{3}\right)^{\lambda}-\left(1-\mu^{3}\right)^{\lambda}\right) /\left(\left(1+\mu^{3}\right)^{\lambda}+\left(1-\mu^{3}\right)^{\lambda}\right)}\right.$,

$$
\left.\left(\sqrt[3]{2} v^{\lambda} / \sqrt[3]{\left(2-\nu^{3}\right)^{\lambda}+\left(\nu^{3}\right)^{\lambda}}\right)\right\rangle
$$

(vii) $\mathscr{R}^{\lambda}=\left\langle\left(\sqrt[3]{2} \mu^{\lambda} / \sqrt[3]{\left(2-\mu^{3}\right)^{\lambda}+\left(\mu^{3}\right)^{\lambda}}\right)\right.$,

$$
\sqrt[3]{\left.\left(\left(1+v^{3}\right)^{\lambda}-\left(1-v^{3}\right)^{\lambda}\right) /\left(\left(1+v^{3}\right)^{\lambda}+\left(1-v^{3}\right)^{\lambda}\right)\right\rangle}
$$

Theorem 1. Let $\mathscr{R}=\left\langle\mu_{\mathscr{R}}, v_{\mathscr{R}}\right\rangle, \mathscr{R}_{1}=\left\langle\mu_{1}, v_{1}\right\rangle$, and $\mathscr{R}_{2}=$ $\left\langle\mu_{2}, v_{2}\right\rangle$ be three FFNs; then, $\mathscr{R}_{3}=\mathscr{R}_{1} \oplus_{\varepsilon} \mathscr{R}_{2}$ and $\mathscr{R}_{4}=\lambda \cdot{ }_{\varepsilon} \mathscr{R}$ are also FFNs.

Proof. Since $\lambda>0$ and $\mathscr{R}$ is an FFN, therefore, $0 \leq \mu_{\mathscr{R}}(x) \leq 1$, $0 \leq \nu_{\mathscr{R}}(x) \leq 1$, and $0 \leq\left(\mu_{\mathscr{R}}(x)\right)^{3}+\left(\nu_{\mathscr{R}}(x)\right)^{3} \leq 1$; then, $1-\left(\mu_{\mathscr{R}}(x)\right)^{3} \geq\left(\nu_{\mathscr{R}}(x)\right)^{3} \geq 0,1-\left(\nu_{\mathscr{R}}(x)\right)^{3} \geq\left(\mu_{\mathscr{R}}(x)\right)^{3} \geq 0$, and $\left(1-\left(\mu_{\mathscr{R}}(x)\right)^{3}\right)^{\lambda} \geq\left(\nu_{\mathscr{R}}(x)\right)^{3}$; then,

$$
\begin{aligned}
& \sqrt[3]{\frac{\left(1+\left(\mu_{\mathscr{R}}(x)\right)^{3}\right)^{\lambda}-\left(1-\left(\mu_{\mathscr{R}}(x)\right)^{3}\right)^{\lambda}}{\left(1+\left(\mu_{\mathscr{R}}(x)\right)^{3}\right)^{\lambda}+\left(1-\left(\mu_{\mathscr{R}}(x)\right)^{3}\right)^{\lambda}}} \\
& \leq \sqrt[3]{\frac{\left(1+\left(\mu_{\mathscr{R}}(x)\right)^{3}\right)^{\lambda}-\left(\left(\nu_{\mathscr{R}}(x)\right)^{3}\right)^{\lambda}}{\left(1+\left(\mu_{\mathscr{R}}(x)\right)^{3}\right)^{\lambda}+\left(\left(\nu_{\mathscr{R}}(x)\right)^{3}\right)^{\lambda}}} \\
& \sqrt[3]{\left(2-\left(v_{\mathscr{R}}(x)\right)^{3}\right)^{\lambda}+\left(v_{\mathscr{R}}(x)^{3}\right)^{\lambda}} \\
& \leq \frac{\sqrt[3]{2}\left(v_{\mathscr{R}}(x)\right)^{\lambda}}{\sqrt[3]{\left(1+\left(\nu_{\mathscr{R}}(x)\right)^{3}\right)^{\lambda}+\left(\left(\nu_{\mathscr{R}}(x)\right)^{3}\right)^{\lambda}}}
\end{aligned}
$$

Thus,

$$
\begin{aligned}
& \left(\sqrt[3]{\frac{\left(1+\left(\mu_{\mathscr{R}}(x)\right)^{3}\right)^{\lambda}-\left(1-\left(\mu_{\mathscr{R}}(x)\right)^{3}\right)^{\lambda}}{\left(1+\left(\mu_{\mathscr{R}}(x)\right)^{3}\right)^{\lambda}+\left(1-\left(\mu_{\mathscr{R}}(x)\right)^{3}\right)^{\lambda}}}\right)^{3} \\
& +\left(\frac{\sqrt[3]{2}\left(\nu_{\mathscr{R}}(x)\right)^{\lambda}}{\sqrt[3]{\left(2-\left(v_{\mathscr{R}}(x)\right)^{3}\right)^{\lambda}+\left(\left(\nu_{\mathscr{R}}(x)\right)^{3}\right)^{\lambda}}}\right)^{3} \leq 1 .
\end{aligned}
$$

Furthermore,

$$
\begin{aligned}
& \left(\sqrt[3]{\frac{\left(1+\left(\mu_{\mathscr{R}}(x)\right)^{3}\right)^{\lambda}-\left(1-\left(\mu_{\mathscr{R}}(x)\right)^{3}\right)^{\lambda}}{\left(1+\left(\mu_{\mathscr{R}}(x)\right)^{3}\right)^{\lambda}+\left(1-\left(\mu_{\mathscr{R}}(x)\right)^{3}\right)^{\lambda}}}\right)^{3} \\
& +\left(\frac{\sqrt[3]{2}\left(\nu_{\mathscr{R}}(x)\right)^{\lambda}}{\sqrt[3]{\left(2-\left(v_{\mathscr{R}}(x)\right)^{3}\right)^{\lambda}+\left(v_{\mathscr{R}}(x)^{3}\right)^{\lambda}}}\right)^{3}=0,
\end{aligned}
$$

iff $\mu_{\mathscr{R}}(x)=\nu_{\mathscr{R}}(x)=0$ and

$$
\begin{aligned}
& \left(\sqrt[3]{\left.\frac{\left(1+\left(\mu_{\mathscr{R}}(x)\right)^{3}\right)^{\lambda}-\left(1-\left(\mu_{\mathscr{R}}(x)\right)^{3}\right)^{\lambda}}{\left(1+\left(\mu_{\mathscr{R}}(x)\right)^{3}\right)^{\lambda}+\left(1-\left(\mu_{\mathscr{R}}(x)\right)^{3}\right)^{\lambda}}\right)^{3}}\right)^{3}=1, \\
& +\left(\frac{\sqrt[3]{2}\left(\nu_{\mathscr{R}}(x)\right)^{\lambda}}{\sqrt[3]{\left(2-\left(\nu_{\mathscr{R}}(x)\right)^{3}\right)^{\lambda}+\left(v_{\mathscr{R}}(x)^{3}\right)^{\lambda}}}\right)^{3}
\end{aligned}
$$

iff $\left(\mu_{\mathscr{R}}(x)\right)^{3}+\left(\nu_{\mathscr{R}}(x)\right)^{3}=1$.

Thus, $\mathscr{R}_{4}=\lambda \cdot{ }_{\varepsilon} \mathscr{R}$ is an FFN for $\lambda>0$.

Theorem 2. Let $\lambda, \lambda_{1}, \lambda_{2} \geq 0$; then,

(i) $\mathscr{R}_{1} \oplus_{\varepsilon} \mathscr{R}_{2}=\mathscr{R}_{2} \oplus_{\varepsilon} \mathscr{R}_{1}$

(ii) $\mathscr{R}_{1} \otimes_{\varepsilon} \mathscr{R}_{2}=\mathscr{R}_{1} \otimes_{\varepsilon} \mathscr{R}_{2}$

(iii) $\lambda \cdot{ }_{\varepsilon}\left(\mathscr{R}_{1} \oplus_{\varepsilon} \mathscr{R}_{2}\right)=\lambda \cdot \mathscr{R}_{\varepsilon} \oplus_{\varepsilon} \lambda \cdot{ }_{\varepsilon} \mathscr{R}_{2}$ 
(iv) $\left(\mathscr{R}_{1} \otimes_{\varepsilon} \mathscr{R}_{2}\right)^{\lambda}=\mathscr{R}_{1}^{\lambda} \otimes_{\varepsilon} \mathscr{R}_{2}^{\lambda}$

(v) $\lambda_{1} \cdot{ }_{\varepsilon} \mathscr{R} \oplus_{\varepsilon} \lambda_{2} \cdot{ }_{\varepsilon} \mathscr{R}=\left(\lambda_{1}+\lambda_{2}\right) \cdot{ }_{\varepsilon} \mathscr{R}$

(vi) $\mathscr{R}^{\lambda_{1}} \otimes_{\varepsilon} \mathscr{R}^{\lambda_{2}}=\mathscr{R}^{\left(\lambda_{1}+\lambda_{2}\right)}$

\section{Proof}

(i)

$$
\begin{aligned}
\mathscr{R}_{1} \oplus_{\varepsilon} \mathscr{R}_{2} & =\left\langle\sqrt[3]{\frac{\mu_{1}^{3}+\mu_{2}^{3}}{1+\mu_{1}^{3} \cdot{ }_{\varepsilon} \mu_{2}^{3}}}, \frac{v_{1} \cdot{ }_{\varepsilon} v_{2}}{\sqrt[3]{1+\left(1-v_{1}^{3}\right) \cdot \varepsilon\left(1-v_{2}^{3}\right)}}\right\rangle \\
& =\left\langle\sqrt[3]{\frac{\mu_{2}^{3}+\mu_{1}^{3}}{1+\mu_{2}^{3} \cdot{ }_{\varepsilon} \mu_{1}^{3}}}, \frac{v_{2} \cdot{ }_{\varepsilon} v_{1}}{\sqrt[3]{1+\left(1-v_{2}^{3}\right) \cdot \varepsilon\left(1-v_{1}^{3}\right)}}\right\rangle \\
& =\mathscr{R}_{2} \oplus_{\varepsilon} \mathscr{R}_{1} .
\end{aligned}
$$

(ii)

$$
\mathscr{R}_{1} \oplus_{\varepsilon} \mathscr{R}_{2}=\left\langle\sqrt[3]{\frac{\mu_{1}^{3}+\mu_{2}^{3}}{1+\mu_{1}^{3} \cdot{ }_{\varepsilon} \mu_{2}^{3}}}, \frac{v_{1} \cdot{ }_{\varepsilon} \nu_{2}}{\sqrt[3]{1+\left(1-v_{1}^{3}\right) \cdot \varepsilon\left(1-v_{2}^{3}\right)}}\right\rangle
$$

) is equivalent to

$$
\mathscr{R}_{1} \oplus_{\varepsilon} \mathscr{R}_{2}=\left\langle\sqrt[3]{\frac{\left(1+\mu_{1}^{3}\right) \cdot{ }_{\varepsilon}\left(1+\mu_{2}^{3}\right)-\left(1-\mu_{1}^{3}\right) \cdot{ }_{\varepsilon}\left(1-\mu_{2}^{3}\right)}{\left(1+\mu_{1}^{3}\right) \cdot{ }_{\varepsilon}\left(1+\mu_{2}^{3}\right)+\left(1-\mu_{1}^{3}\right) \cdot{ }_{\varepsilon}\left(1-\mu_{2}^{3}\right)}},\right.
$$

$$
\left.\frac{\sqrt[3]{2} v_{1} \cdot v_{2}}{\sqrt[3]{\left(2-v_{1}^{3}\right) \cdot \varepsilon\left(2-v_{2}^{3}\right)+v_{1}^{3} \cdot \varepsilon v_{2}^{3}}}\right\rangle
$$

Take $a=\left(1+\mu_{1}^{3}\right) \cdot{ }_{\varepsilon}\left(1+\mu_{2}^{3}\right), \quad b=\left(1-\mu_{1}^{3}\right) \cdot{ }_{\varepsilon}\left(1-\mu_{2}^{3}\right)$, $c=v_{1}^{3} \cdot{ }_{\varepsilon} v_{2}^{3}$, and $d=\left(2-v_{1}^{3}\right) \cdot \varepsilon\left(2-v_{2}^{3}\right)$; then,

$$
\mathscr{R}_{1} \oplus_{\varepsilon} \mathscr{R}_{2}=\left\langle\sqrt[3]{\frac{a-b}{a+b}}, \frac{\sqrt[3]{2 c}}{\sqrt[3]{d+c}}\right\rangle .
$$

By the Einstein FF law, 
where $\quad a_{1}=\left(1+\mu_{1}^{3}\right)^{\lambda}, \quad b_{1}=\left(1-\mu_{1}^{3}\right)^{\lambda}, \quad c_{1}=\left(\nu_{1}^{3}\right)^{\lambda}$, $d_{1}=\left(2-v_{1}^{3}\right)^{\lambda}, \quad a_{2}=\left(1+\mu_{2}^{3}\right)^{\lambda}, \quad b_{2}=\left(1-\mu_{2}^{3}\right)^{\lambda}, \quad c_{2}=\left(\nu_{2}^{3}\right)^{\lambda}$, and $d_{2}=\left(2-v_{2}^{3}\right)^{\lambda}$; therefore,

$$
\begin{aligned}
& \left(\lambda \cdot{ }_{\varepsilon} \mathscr{R}_{1}\right) \oplus_{\varepsilon}\left(\lambda \cdot{ }_{\varepsilon} \mathscr{R}_{2}\right)=\left\langle\sqrt[3]{\frac{a_{1}-b_{1}}{a_{1}+b_{1}}}, \frac{\sqrt[3]{2 c_{1}}}{\sqrt[3]{d_{1}+c_{1}}}\right\rangle \oplus_{\varepsilon}\left\langle\sqrt[3]{\frac{a_{2}-b_{2}}{a_{2}+b_{2}}}, \frac{\sqrt[3]{2 c_{2}}}{\sqrt[3]{d_{2}+c_{2}}}\right\rangle
\end{aligned}
$$

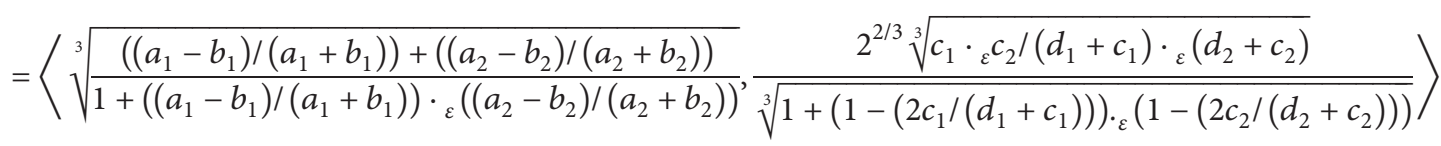

$$
\begin{aligned}
& =\left\langle\sqrt[3]{\frac{a_{1} \cdot{ }_{\varepsilon} a_{2}-b_{1} \cdot{ }_{\varepsilon} b_{2}}{a_{1} \cdot{ }_{\varepsilon} a_{2}+b_{1} \cdot{ }_{\varepsilon} b_{2}}}, \frac{\sqrt[3]{2 c_{1} \cdot{ }_{\varepsilon} c_{2}}}{\sqrt[3]{d_{1} \cdot{ }_{\varepsilon} d_{2}+c_{1} \cdot{ }_{\varepsilon} c_{2}}}\right\rangle \\
& =\left\langle\sqrt[3]{\frac{\left(1+\mu_{1}^{3}\right)^{\lambda} \cdot{ }_{\varepsilon}\left(1+\mu_{2}^{3}\right)^{\lambda}-\left(1-\mu_{1}^{3}\right)^{\lambda} \cdot{ }_{\varepsilon}\left(1-\mu_{2}^{3}\right)^{\lambda}}{\left(1+\mu_{1}^{3}\right)^{\lambda} \cdot{ }_{\varepsilon}\left(1+\mu_{2}^{3}\right)^{\lambda}+\left(1-\mu_{1}^{3}\right)^{\lambda} \cdot{ }_{\varepsilon}\left(1-\mu_{2}^{3}\right)^{\lambda}}}, \frac{\sqrt[3]{2} v_{1}^{\lambda} \cdot \varepsilon_{\varepsilon} v_{2}^{\lambda}}{\sqrt[3]{\left(2-v_{1}^{3}\right)^{\lambda} \cdot \varepsilon\left(2-v_{2}^{3}\right)^{\lambda}+\left(v_{1}^{3}\right)^{\lambda} \cdot{ }_{\varepsilon}\left(v_{2}^{3}\right)^{\lambda}}}\right\rangle .
\end{aligned}
$$

Hence, $\lambda \cdot{ }_{\varepsilon}\left(\mathscr{R}_{1} \oplus_{\varepsilon} \mathscr{R}_{2}\right)=\lambda \cdot{ }_{\varepsilon} \mathscr{R}_{1} \oplus_{\varepsilon} \lambda \cdot{ }_{\varepsilon} \mathscr{R}_{2}$.

(v) For $\lambda_{1}, \lambda_{2}>0$,

$$
\begin{aligned}
& \lambda_{1} \cdot{ }_{\varepsilon} \mathscr{R}=\left\langle\sqrt[3]{\frac{\left(1+\mu^{3}\right)^{\lambda_{1}}-\left(1-\mu^{3}\right)^{\lambda_{1}}}{\left(1+\mu^{3}\right)^{\lambda_{1}}+\left(1-\mu^{3}\right)^{\lambda_{1}}}}, \frac{\sqrt[3]{2} \nu^{\lambda_{1}}}{\sqrt[3]{\left(2-\nu^{3}\right)^{\lambda_{1}}+\left(\nu^{3}\right)^{\lambda_{1}}}}\right\rangle=\left\langle\sqrt[3]{\frac{a_{1}-b_{1}}{a_{1}+b_{1}}}, \frac{\sqrt[3]{2 c_{1}}}{\sqrt[3]{d_{1}+c_{1}}}\right\rangle, \\
& \lambda_{2} \cdot{ }_{\varepsilon} \mathscr{R}=\left\langle\sqrt[3]{\frac{\left(1+\mu^{3}\right)^{\lambda_{2}}-\left(1-\mu^{3}\right)^{\lambda_{2}}}{\left(1+\mu^{3}\right)^{\lambda_{2}}+\left(1-\mu^{3}\right)^{\lambda_{2}}}}, \frac{\sqrt[3]{2} \nu^{\lambda_{2}}}{\sqrt[3]{\left(2-\nu^{3}\right)^{\lambda_{2}}+\left(\nu^{3}\right)^{\lambda_{2}}}}\right\rangle=\left\langle\sqrt[3]{\frac{a_{2}-b_{2}}{a_{2}+b_{2}}}, \frac{\sqrt[3]{2 c_{2}}}{\sqrt[3]{d_{2}+c_{2}}}\right\rangle,
\end{aligned}
$$

where $a_{j}=\left(1+\mu^{3}\right)^{\lambda_{j}}, b_{j}=\left(1-\mu^{3}\right)^{\lambda_{j}}, c_{j}=\left(\nu^{3}\right)^{\lambda_{j}}$, and $d_{j}=\left(2-v^{3}\right)^{\lambda_{j}}$, for $\dot{j}=1,2$.

$$
\begin{aligned}
& \left(\lambda_{1} \cdot{ }_{\varepsilon} \mathscr{R}\right) \oplus_{\varepsilon}\left(\lambda_{2} \cdot{ }_{\varepsilon} \mathscr{R}\right)=\left\langle\sqrt[3]{\frac{a_{1}-b_{1}}{a_{1}+b_{1}}}, \frac{\sqrt[3]{2 c_{1}}}{\sqrt[3]{d_{1}+c_{1}}}\right\rangle \oplus_{\varepsilon}\left\langle\sqrt[3]{\frac{a_{2}-b_{2}}{a_{2}+b_{2}}}, \frac{\sqrt[3]{2 c_{2}}}{\sqrt[3]{d_{2}+c_{2}}}\right\rangle \\
& =\left\langle\sqrt[3]{\frac{\left(\left(a_{1}-b_{1}\right) /\left(a_{1}+b_{1}\right)\right)+\left(\left(a_{2}-b_{2}\right) /\left(a_{2}+b_{2}\right)\right)}{1+\left(\left(a_{1}-b_{1}\right) /\left(a_{1}+b_{1}\right)\right) \cdot{ }_{\varepsilon}\left(\left(a_{2}-b_{2}\right) /\left(a_{2}+b_{2}\right)\right)}}, \frac{2^{2 / 3} \sqrt[3]{c_{1} \cdot{ }_{\varepsilon} c_{2} /\left(d_{1}+c_{1}\right) \cdot{ }_{\varepsilon}\left(d_{2}+c_{2}\right)}}{\sqrt[3]{1+\left(1-\left(2 c_{1} /\left(d_{1}+c_{1}\right)\right)\right) \cdot{ }_{\varepsilon}\left(1-\left(2 c_{2} /\left(d_{2}+c_{2}\right)\right)\right)}}\right\rangle \\
& =\left\langle\sqrt[3]{\frac{a_{1} \cdot{ }_{\varepsilon} a_{2}-b_{1} \cdot{ }_{\varepsilon} b_{2}}{a_{1} \cdot{ }_{\varepsilon} a_{2}+b_{1} \cdot{ }_{\varepsilon} b_{2}}}, \frac{\sqrt[3]{2 c_{1} \cdot{ }_{\varepsilon} c_{2}}}{\sqrt[3]{d_{1} \cdot{ }_{\varepsilon} d_{2}+c_{1} \cdot{ }_{\varepsilon} c_{2}}}\right\rangle \\
& =\left\langle\sqrt[3]{\frac{\left(1+\mu^{3}\right)^{\lambda_{1}+\lambda_{2}}-\left(1-\mu^{3}\right)^{\lambda_{1}+\lambda_{2}}}{\left(1+\mu^{3}\right)^{\lambda_{1}+\lambda_{2}}+\left(1-\mu^{3}\right)^{\lambda_{1}+\lambda_{2}}}}, \frac{\sqrt[3]{\left(2-\nu^{3}\right)^{\lambda_{1}+\lambda_{2}}+\left(\nu_{1}^{3}\right)^{\lambda_{1}+\lambda_{2}}}}{\sqrt[3]{\lambda_{1}+\lambda_{2}}}\right\rangle \\
& =\left(\lambda_{1}+\lambda_{2}\right) \cdot{ }_{\varepsilon} \mathscr{R} .
\end{aligned}
$$


Hence, $\lambda_{1} \cdot{ }_{\varepsilon} \mathscr{R} \oplus_{\varepsilon} \lambda_{2} \cdot \varepsilon_{\varepsilon} \mathscr{R}=\left(\lambda_{1}+\lambda_{2}\right) \cdot{ }_{\varepsilon} \mathscr{R}$.

Similarly, others can be verified.

Theorem 3. Let $\mathscr{R}_{1}=\left\langle\mu_{1}, v_{1}\right\rangle$ and $\mathscr{R}_{2}=\left\langle\mu_{2}, v_{2}\right\rangle$ be FFNs; then,
(i) $\mathscr{R}_{1}^{c} \wedge_{\varepsilon} \mathscr{R}_{2}^{c}=\left(\mathscr{R}_{1} \vee_{\varepsilon} \mathscr{R}_{2}\right)^{c}$
(ii) $\mathscr{R}_{1}^{c} \vee_{\varepsilon} \mathscr{R}_{2}^{c}=\left(\mathscr{R}_{1} \wedge_{\varepsilon} \mathscr{R}_{2}\right)^{c}$
(iii) $\mathscr{R}_{1}^{c} \oplus_{\varepsilon} \mathscr{R}_{2}^{c}=\left(\mathscr{R}_{1} \otimes_{\varepsilon} \mathscr{R}_{2}\right)^{c}$
(iv) $\mathscr{R}_{1}^{c} \otimes_{\varepsilon} \mathscr{R}_{2}^{c}=\left(\mathscr{R}_{1} \oplus_{\varepsilon} \mathscr{R}_{2}\right)^{c}$
(v) $\left(\mathscr{R}_{1} \vee_{\varepsilon} \mathscr{R}_{2}\right) \oplus_{\varepsilon}\left(\mathscr{R}_{1} \wedge_{\varepsilon} \mathscr{R}_{2}\right)=\mathscr{R}_{1} \oplus_{\varepsilon} \mathscr{R}_{2}$
(vi) $\left(\mathscr{R}_{1} \vee_{\varepsilon} \mathscr{R}_{2}\right) \oplus_{\varepsilon}\left(\mathscr{R}_{1} \wedge_{\varepsilon} \mathscr{R}_{2}\right)=\mathscr{R}_{1} \oplus_{\varepsilon} \mathscr{R}_{2}$

Proof. It is obvious.

Theorem 4. Let $\mathscr{R}_{1}=\left\langle\mu_{1}, v_{1}\right\rangle, \mathscr{R}_{2}=\left\langle\mu_{2}, v_{2}\right\rangle$, and $\mathscr{R}_{3}=$ $\left\langle\mu_{3}, v_{3}\right\rangle$ be three FFNs; then,

(i) $\left(\mathscr{R}_{1} \vee_{\varepsilon} \mathscr{R}_{2}\right) \wedge_{\varepsilon} \mathscr{R}_{3}=\left(\mathscr{R}_{1} \wedge_{\varepsilon} \mathscr{R}_{3}\right) \vee_{\varepsilon}\left(\mathscr{R}_{2} \wedge_{\varepsilon} \mathscr{R}_{3}\right)$

(ii) $\left(\mathscr{R}_{1} \wedge_{\varepsilon} \mathscr{R}_{2}\right) \vee_{\varepsilon} \mathscr{R}_{3}=\left(\mathscr{R}_{1} \vee_{\varepsilon} \mathscr{R}_{3}\right) \wedge_{\varepsilon}\left(\mathscr{R}_{2} \vee_{\varepsilon} \mathscr{R}_{3}\right)$

(iii) $\left(\mathscr{R}_{1} \vee_{\varepsilon} \mathscr{R}_{2}\right) \oplus_{\varepsilon} \mathscr{R}_{3}=\left(\mathscr{R}_{1} \oplus_{\varepsilon} \mathscr{R}_{3}\right) \vee_{\varepsilon}\left(\mathscr{R}_{2} \oplus_{\varepsilon} \mathscr{R}_{3}\right)$

(iv) $\left(\mathscr{R}_{1} \wedge_{\varepsilon} \mathscr{R}_{2}\right) \oplus_{\varepsilon} \mathscr{R}_{3}=\left(\mathscr{R}_{1} \oplus_{\varepsilon} \mathscr{R}_{3}\right) \wedge_{\varepsilon}\left(\mathscr{R}_{2} \oplus_{\varepsilon} \mathscr{R}_{3}\right)$

(v) $\left(\mathscr{R}_{1} \vee_{\varepsilon} \mathscr{R}_{2}\right) \otimes_{\varepsilon} \mathscr{R}_{3}=\left(\mathscr{R}_{1} \otimes_{\varepsilon} \mathscr{R}_{3}\right) \vee_{\varepsilon}\left(\mathscr{R}_{2} \otimes_{\varepsilon} \mathscr{R}_{3}\right)$

(vi) $\left(\mathscr{R}_{1} \wedge_{\varepsilon} \mathscr{R}_{2}\right) \otimes_{\varepsilon} \mathscr{R}_{3}=\left(\mathscr{R}_{1} \otimes_{\varepsilon} \mathscr{R}_{3}\right) \wedge_{\varepsilon}\left(\mathscr{R}_{2} \otimes_{\varepsilon} \mathscr{R}_{3}\right)$
Proof. The proof is trivial, so we omit it.

\section{Fermatean Fuzzy Einstein Weighted Averaging Operators}

The Einstein weighted averaging operators under FF environment are defined here.

Definition 7. Let $\mathscr{R}_{j}=\left\langle\mu_{j}, v_{j}\right\rangle(j=1,2, \ldots, s)$ be a collection of FFNs and $w_{j}$ be the weight vector (WV) of $\mathscr{R}_{j}$ with $w_{j}>0$ and $\sum_{j=1}^{j} w_{j}=1$; then, FFEWA operator is a mapping $\mathbb{Q}^{s} \longrightarrow Q_{Q}$ such that

$\operatorname{FFEWA}\left(\mathscr{R}_{1}, \mathscr{R}_{2}, \ldots, \mathscr{R}_{j}\right)=w_{1} \cdot{ }_{\varepsilon} \mathscr{R}_{1} \oplus_{\varepsilon} w_{2} \cdot{ }_{\varepsilon} \mathscr{R}_{2} \oplus_{\varepsilon} \cdots \oplus_{\varepsilon} w_{\jmath} \cdot{ }_{\varepsilon} \mathscr{R}_{j}$.

If $w_{j}=(1 / s), \forall j$, then FFEWA operator becomes FFWA operator:

$$
\operatorname{FFA}\left(\mathscr{R}_{1}, \mathscr{R}_{2}, \ldots, \mathscr{R}_{3}\right)=\frac{1}{s}\left(\mathscr{R}_{1} \oplus_{\varepsilon} \mathscr{R}_{2} \oplus_{\varepsilon} \cdots \oplus_{\varepsilon} \mathscr{R}_{3}\right) .
$$

Theorem 5. Let $\mathscr{R}_{j}=\left(\mu_{j}, \nu_{j}\right)$ be FFNs; then, the aggregated value by using equation (18) is

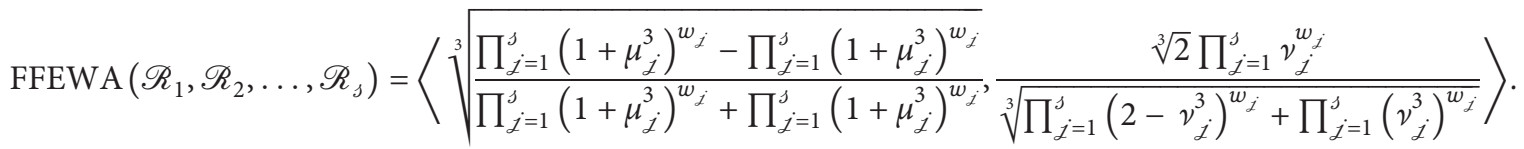

Proof. Use the mathematical induction to prove equation (20).

When $s=2$,

$$
\operatorname{FFEWA}\left(\mathscr{R}_{1}, \mathscr{R}_{2}\right)=w_{1} \cdot{ }_{\varepsilon} \mathscr{R}_{1} \oplus_{\varepsilon} w_{2} \cdot{ }_{\varepsilon} \mathscr{R}_{2} .
$$

By Theorem 1, both $w_{1} \cdot{ }_{\varepsilon} \mathscr{R}_{1}$ and $w_{2} \cdot \mathscr{R}_{\varepsilon}$ are FFNs and value of $w_{1} \cdot \mathscr{R}_{\varepsilon} \oplus_{\varepsilon} w_{2} \cdot \mathscr{R}_{2}$ is an FFN. By using (vi) in Definition 6,

$$
\begin{aligned}
& w_{1} \cdot{ }_{\varepsilon} \mathscr{R}_{1}=\left\langle\sqrt[3]{\frac{\left(1+\mu_{1}^{3}\right)^{w_{1}}-\left(1-\mu_{1}^{3}\right)^{w_{1}}}{\left(1+\mu_{1}^{3}\right)^{w_{1}}+\left(1-\mu_{1}^{3}\right)^{w_{1}}}}, \frac{\sqrt[3]{2 \nu_{1}^{w_{1}}}}{\sqrt[3]{\left(2-v_{1}^{3}\right)^{w_{1}}+\left(v_{1}^{3}\right)^{w_{1}}}}\right\rangle, \\
& w_{2} \cdot{ }_{\varepsilon} \mathscr{R}_{2}=\left\langle\sqrt[3]{\frac{\left(1+\mu_{2}^{3}\right)^{w_{2}}-\left(1-\mu_{2}^{3}\right)^{w_{2}}}{\left(1+\mu_{2}^{3}\right)^{w_{2}}+\left(1-\mu_{2}^{3}\right)^{w_{2}}}}, \frac{\sqrt[3]{2} v_{2}^{w_{2}}}{\sqrt[3]{\left(2-v_{2}^{3}\right)^{w_{2}}+\left(v_{2}^{3}\right)^{w_{2}}}}\right\rangle .
\end{aligned}
$$


Then,

$\operatorname{FFEWA}\left(\mathscr{R}_{1}, \mathscr{R}_{2}\right)=w_{1} \cdot{ }_{\varepsilon} \mathscr{R}_{1} \oplus_{\varepsilon} w_{2} \cdot{ }_{\varepsilon} \mathscr{R}_{2}$

$$
\begin{aligned}
& =\left\langle\sqrt[3]{\frac{\left(\left(\left(1+\mu_{1}^{3}\right)^{w_{1}}-\left(1-\mu_{1}^{3}\right)^{w_{1}}\right) /\left(\left(1+\mu_{1}^{3}\right)^{w_{1}}+\left(1-\mu_{1}^{3}\right)^{w_{1}}\right)\right)+\left(\left(\left(1+\mu_{2}^{3}\right)^{w_{2}}-\left(1-\mu_{2}^{3}\right)^{w_{2}}\right) /\left(\left(1+\mu_{2}^{3}\right)^{w_{2}}+\left(1-\mu_{2}^{3}\right)^{w_{2}}\right)\right)}{1+\left(\left(\left(1+\mu_{1}^{3}\right)^{w_{1}}-\left(1-\mu_{1}^{3}\right)^{w_{1}}\right) /\left(\left(1+\mu_{1}^{3}\right)^{w_{1}}+\left(1-\mu_{1}^{3}\right)^{w_{1}}\right)\right) \cdot{ }_{\varepsilon}\left(\left(\left(1+\mu_{2}^{3}\right)^{w_{2}}-\left(1-\mu_{2}^{3}\right)^{w_{2}}\right) /\left(\left(1+\mu_{2}^{3}\right)^{w_{2}}+\left(1-\mu_{2}^{3}\right)^{w_{2}}\right)\right)}},\right. \\
& \left.\frac{\left(\sqrt[3]{2} v_{1}^{w_{1}} / \sqrt[3]{\left(2-v_{1}^{3}\right)^{w_{1}}+\left(v_{1}^{3}\right)^{w_{1}}}\right) \cdot{ }_{\varepsilon}\left(\sqrt[3]{2} \nu_{1}^{w_{2}} / \sqrt[3]{\left(2-v_{2}^{3}\right)^{w_{2}}+\left(v_{2}^{3}\right)^{w_{2}}}\right)}{\sqrt[3]{1+\left(1-\left(2 v_{1}^{3 w_{1}} /\left(\left(2-v_{1}^{3}\right)^{w_{1}}+\left(v_{1}^{3}\right)^{w_{1}}\right)\right)\right) \cdot{ }_{\varepsilon}\left(1-\left(2 v_{2}^{3 w_{2}} /\left(\left(2-v_{2}^{3}\right)^{w_{2}}+\left(v_{2}^{3}\right)^{w_{2}}\right)\right)\right)}}\right\rangle \\
& =\left\langle\sqrt[3]{\frac{\left(1+\mu_{1}^{3}\right)^{w_{1}} \cdot{ }_{\varepsilon}\left(1+\mu_{2}^{3}\right)^{w_{2}}-\left(1-\mu_{1}^{3}\right)^{w_{1}} \cdot \varepsilon_{\varepsilon}\left(1-\mu_{2}^{3}\right)^{w_{2}}}{\left(1+\mu_{1}^{3}\right)^{w_{1}} \cdot \varepsilon_{\varepsilon}\left(1+\mu_{2}^{3}\right)^{w_{2}}+\left(1-\mu_{1}^{3}\right)^{w_{1}} \cdot{ }_{\varepsilon}\left(1-\mu_{2}^{3}\right)^{w_{2}}}}, \frac{\sqrt[3]{2} \nu_{1}^{w_{1}} \cdot{ }_{\varepsilon} v_{2}^{w_{2}}}{\sqrt[3]{\left(2-\nu_{1}^{3}\right)^{w_{1}} \cdot \varepsilon\left(2-v_{2}^{3}\right)^{w_{2}}+\left(\nu_{1}^{3}\right)^{w_{1}} \cdot{ }_{\varepsilon}\left(\nu_{2}^{3}\right)^{w_{2}}}}\right\rangle .
\end{aligned}
$$

Thus, equation (20) is true when $s=2$.

Suppose result is true for $s=k$ :

$$
\operatorname{FFEWA}\left(\mathscr{R}_{1}, \mathscr{R}_{2}, \ldots, \mathscr{R}_{k}\right)=\left\langle\sqrt[3]{\frac{\prod_{j=1}^{k}\left(1+\mu_{j}^{3}\right)^{w_{j}}-\prod_{j=1}^{k}\left(1-\mu_{j}^{3}\right)^{w_{j}}}{\prod_{j=1}^{k}\left(1+\mu_{j}^{3}\right)^{w_{j}}+\prod_{j=1}^{k}\left(1-\mu_{j}^{3}\right)^{w_{j}}}}, \frac{\sqrt[3]{2} \prod_{j=1}^{k} v_{j}^{w_{j}}}{\sqrt[3]{\prod_{j=1}^{k}\left(2-v_{j}^{3}\right)^{w_{j}}+\prod_{j=1}^{k}\left(v_{j}^{3}\right)^{w_{j}}}}\right\rangle .
$$

Now, for $s=k+1$,

$$
\begin{aligned}
& \operatorname{FFEWA}\left(\mathscr{R}_{1}, \mathscr{R}_{2}, \ldots, \mathscr{R}_{k+1}\right)=\left\langle\sqrt[3]{\frac{\prod_{j=1}^{k}\left(1+\mu_{j}^{3}\right)^{w_{j}}-\prod_{j=1}^{k}\left(1-\mu_{j}^{3}\right)^{w_{j}}}{\prod_{j=1}^{k}\left(1+\mu_{j}^{3}\right)^{w_{j}}+\prod_{j=1}^{k}\left(1-\mu_{j}^{3}\right)^{w_{j}}}}, \frac{\sqrt[3]{2} \prod_{j=1}^{k} v_{j}^{w_{j}}}{\sqrt[\prod_{j=1}^{k}\left(2-v_{j}^{3}\right)^{w_{j}}+\prod_{j=1}^{k}\left(v_{j}^{3}\right)^{w_{j}}]{\prod_{j}}}\right\rangle \\
& \oplus_{\varepsilon}\left\langle\sqrt[3]{\frac{\left(1+\mu_{k+1}^{3}\right)^{w_{k+1}}-\left(1-\mu_{k+1}^{3}\right)^{w_{k+1}}}{\left(1+\mu_{k+1}^{3}\right)^{w_{k+1}}+\left(1-\mu_{k+1}^{3}\right)^{w_{k+1}}}}, \frac{\sqrt[3]{2} v_{k+1}^{w_{k+1}}}{\sqrt[3]{\left(2-v_{k+1}^{3}\right)^{w_{k+1}}+\left(v_{k+1}^{3}\right)^{w_{k+1}}}}\right\rangle \\
& =\left\langle\sqrt[3]{\frac{\prod_{j=1}^{k+1}\left(1+\mu_{j}^{3}\right)^{w_{j}}-\prod_{j=1}^{k+1}\left(1-\mu_{j}^{3}\right)^{w_{j}}}{\prod_{j=1}^{k+1}\left(1+\mu_{j}^{3}\right)^{w_{j}}+\prod_{j=1}^{k+1}\left(1-\mu_{j}^{3}\right)^{w_{j}}}}, \frac{\sqrt[3]{2} \prod_{j=1}^{k+1} \nu_{j}^{w_{j}}}{\sqrt[3]{\prod_{j=1}^{k+1}\left(2-v_{j}^{3}\right)^{w_{j}}+\prod_{j=1}^{k+1}\left(v_{j}^{3}\right)^{w_{j}}}}\right\rangle .
\end{aligned}
$$

Thus, the result is true for $s=k+1$. Hence, equation where equality holds iff $R_{1}=R_{2}=\cdots=R_{s}$. (20) holds, $\forall s$.

Lemma 1. Let $\left.\mathscr{R}_{j}=\left\langle\mu_{j}, v_{j}\right\rangle, w_{j}\right\rangle 0$, and $\sum_{j=1}^{j} w_{j}=1$; then,

$$
\prod_{j=1}^{s} R_{j}^{w_{j}} \leq \sum_{j=1}^{s} w_{j} R_{j}
$$

Theorem 6. If $\mathscr{R}_{j}=\left\langle\mu_{j}, v_{j}\right\rangle$ are FFNs, then FFEWA $\left(\mathscr{R}_{1}, \mathscr{R}_{2}, \ldots, \mathscr{R}_{3}\right)$ is also an FFN.

Proof. Since $\mathscr{R}_{j}=\left\langle\mu_{j}, v_{j}\right\rangle$ are FFNs, so $0 \leq \mu_{j}, v_{j} \leq 1$ and $0 \leq \mu_{j}^{3}+v_{j}^{3} \leq 1$. Therefore, 


$$
\begin{aligned}
& \frac{\prod_{j=1}^{s}\left(1+\mu_{j}^{3}\right)^{w_{j}}-\prod_{j=1}^{s}\left(1-\mu_{j}^{3}\right)^{w_{j}}}{\prod_{j=1}^{s}\left(1+\mu_{j}^{3}\right)^{w_{j}}+\prod_{j=1}^{s}\left(1-\mu_{j}^{3}\right)^{w_{j}}}=1 \\
& \quad-\frac{2 \prod_{j=1}^{s}\left(1-\mu_{j}^{3}\right)^{w_{j}}}{\prod_{j=1}^{s}\left(1+\mu_{j}^{3}\right)^{w_{j}}+\prod_{j=1}^{s}\left(1-\mu_{j}^{3}\right)^{w_{j}}} \\
& \quad \leq 1-\prod_{j=1}^{s}\left(1-\mu_{j}^{3}\right)^{w_{j}} \leq 1 .
\end{aligned}
$$

Also, $\quad\left(1+\mu_{j}^{3}\right) \geq\left(1-\mu_{j}^{3}\right) \Rightarrow \prod_{j=1}^{s}\left(1+\mu_{j}^{3}\right)-\prod_{j=1}^{s}$ $\left(1-\mu_{j}^{3}\right) \geq 0$. Therefore,

$$
\frac{\prod_{j=1}^{s}\left(1+\mu_{j}^{3}\right)^{w_{j}}-\prod_{j=1}^{s}\left(1-\mu_{j}^{3}\right)^{w_{j}}}{\prod_{j=1}^{s}\left(1+\mu_{j}^{3}\right)^{w_{j}}+\prod_{j=1}^{s}\left(1-\mu_{j}^{3}\right)^{w_{j}}} \geq 0 .
$$

Thus, $0 \leq \mu_{\text {FFEWA }} \leq 1$.

Moreover,

$$
\begin{aligned}
& \frac{2 \prod_{j=1}^{s}\left(v_{j}^{3}\right)^{w_{j}}}{\prod_{j=1}^{s}\left(2-v_{j}^{3}\right)^{w_{j}}+\prod_{j=1}^{s}\left(v_{j}^{3}\right)^{w_{j}}} \\
& \leq \frac{2 \prod_{j=1}^{s}\left(1-\mu_{j}^{3}\right)^{w_{j}}}{\prod_{j=1}^{s}\left(1+\mu_{j}^{3}\right)^{w_{j}}+\prod_{j=1}^{j}\left(1-\mu_{j}^{3}\right)^{w_{j}}} \\
& \leq \prod_{j=1}^{j}\left(1-\mu_{j}^{3}\right)^{w_{j}} \leq 1 .
\end{aligned}
$$

Also,

$$
\prod_{j=1}^{s}\left(v_{j}^{3}\right)^{w_{j}} \geq 0 \Longleftrightarrow \frac{2 \prod_{j=1}^{s}\left(v_{j}^{3}\right)^{w_{j}}}{\prod_{j=1}^{s}\left(2-v_{j}^{3}\right)^{w_{j}}+\prod_{j=1}^{s}\left(v_{j}^{3}\right)^{w_{j}}} \geq 0 .
$$

Thus, $0 \leq \nu_{\text {FFEWA }} \leq 1$. Moreover,

$$
\begin{aligned}
\mu_{\mathrm{FFEWA}}^{3}+v_{\mathrm{FFEWA}}^{3} & =\frac{\prod_{j=1}^{s}\left(1+\mu_{j}^{3}\right)^{w_{j}}-\prod_{j=1}^{s}\left(1-\mu_{j}^{3}\right)^{w_{j}}}{\prod_{j=1}^{s}\left(1+\mu_{j}^{3}\right)^{w_{j}}+\prod_{j=1}^{s}\left(1-\mu_{j}^{3}\right)^{w_{j}}}+\frac{2 \prod_{j=1}^{j}\left(v_{j}^{3}\right)^{w_{j}}}{\prod_{j=1}^{s}\left(2-v_{j}^{3}\right)^{w_{j}}+\prod_{j=1}^{s}\left(v_{j}^{3}\right)^{w_{j}}} \\
& \leq 1-\frac{2 \prod_{j=1}^{s}\left(1-\mu_{j}^{3}\right)^{w_{j}}}{\prod_{j=1}^{s}\left(1+\mu_{j}^{3}\right)^{w_{j}}+\prod_{j=1}^{s}\left(1-\mu_{j}^{3}\right)^{w_{j}}}+\frac{2 \prod_{j=1}^{s}\left(1-\mu_{j}^{3}\right)^{w_{j}}}{\prod_{j=1}^{s}\left(1+\mu_{j}^{3}\right)^{w_{j}}+\prod_{j=1}^{j}\left(1-\mu_{j}^{3}\right)^{w_{j}}} \\
& =1 .
\end{aligned}
$$

Hence, FFEWA $\in[0,1]$. Therefore, FFEWA $\left(\mathscr{R}_{1}, \mathscr{R}_{2}, \ldots, \mathscr{R}_{\jmath}\right) \in$ FFN

$$
\begin{aligned}
& \sqrt[3]{\frac{\prod_{j=1}^{s}\left(1+\mu_{j}^{3}\right)^{w_{j}}-\prod_{j=1}^{s}\left(1-\mu_{j}^{3}\right)^{w_{j}}}{\prod_{j=1}^{s}\left(1+\mu_{j}^{3}\right)^{w_{j}}+\prod_{j=1}^{s}\left(1-\mu_{j}^{3}\right)^{w_{j}}}} \\
& \leq \sqrt[3]{1-\prod_{j=1}^{s}\left(1-\mu_{j}^{3}\right)^{w_{j}} \Longleftrightarrow \mu_{\mathscr{R}}^{\beta} \leq \mu_{\mathscr{R}}},
\end{aligned}
$$

Corollary 1. The FFEWA and FFWA operators have the relationship:

$$
\operatorname{FFEWA}\left(\mathscr{R}_{1}, \mathscr{R}_{2}, \ldots, \mathscr{R}_{\jmath}\right) \leq \operatorname{FFWA}\left(\mathscr{R}_{1}, \mathscr{R}_{2}, \ldots, \mathscr{R}_{\jmath}\right) .
$$

Proof. Let FFEWA $\left(\mathscr{R}_{1}, \mathscr{R}_{2}, \ldots, \mathscr{R}_{j}\right)=\left(\mu_{\mathscr{R}}^{\beta}, \nu_{\mathscr{R}}^{\beta}\right)=\mathscr{R}^{\beta}$

and FFWA $\left(\mathscr{R}_{1}, \mathscr{R}_{2}, \ldots, \mathscr{R}_{1}\right)=\left(\mu_{\mathscr{R}}, v_{\mathscr{R}}\right)=\mathscr{R}$. Since $\prod_{j=1}^{j}\left(1+\mu_{j}^{3}\right)^{w_{j}}+\prod_{j=1}^{j}\left(1-\mu_{j}^{3}\right)^{w_{j}} \leq \sum_{j=1}^{j}\left(1+\mu_{j}^{3}\right)^{w_{j}}+$ $\sum_{j=1}^{j}\left(1-\mu_{j}^{3}\right)^{w_{j}}=2$, then from equation (27), we obtain

equality holds iff $\mu_{1}=\mu_{2}=\cdots=\mu_{\jmath}$.

Also,

$$
\begin{aligned}
& \frac{2 \prod_{j=1}^{s}\left(v_{j}^{3}\right)^{w_{j}}}{\prod_{j=1}^{s}\left(2-v_{j}^{3}\right)^{w_{j}}+\prod_{j=1}^{s}\left(v_{j}^{3}\right)^{w_{j}}} \geq \frac{2 \prod_{j=1}^{s}\left(v_{j}^{3}\right)^{w_{j}}}{\sum_{j=1}^{s} w_{j}\left(2-v_{j}^{3}\right)+\sum_{j=1}^{s} w_{j} v_{j}^{3}} \\
& \geq \prod_{j=1}^{s}\left(v_{j}^{3}\right)^{w_{j}} \Rightarrow \sqrt[3]{\frac{2 \prod_{j=1}^{s}\left(v_{j}^{3}\right)^{w_{j}}}{\prod_{j=1}^{s}\left(2-v_{j}^{3}\right)^{w_{j}}+\prod_{j=1}^{s}\left(v_{j}^{3}\right)^{w_{j}}}} \geq \prod_{j=1}^{s} v_{j}^{w_{j}} \Rightarrow v_{\mathscr{R}}^{\beta} \leq v_{\mathscr{R}},
\end{aligned}
$$


equality holds iff $v_{1}=v_{2}=\cdots=v_{s}$.

Thus,

$$
\mathcal{S}\left(\mathscr{R}^{\beta}\right)=\left(\mu_{\mathscr{R}}^{\beta}\right)^{3}-\left(\nu_{\mathscr{R}}^{\beta}\right)^{3} \leq\left(\mu_{\mathscr{R}}\right)^{3}-\left(\nu_{\mathscr{R}}\right)^{3}=\mathcal{S}(\mathscr{R}) .
$$

If $\mathcal{S}\left(\mathscr{R}^{\beta}\right)<\mathcal{S}(\mathscr{R})$, then

$\operatorname{FFEWA}\left(\mathscr{R}_{1}, \mathscr{R}_{2}, \ldots, \mathscr{R}_{3}\right)<\operatorname{FFWA}\left(\mathscr{R}_{1}, \mathscr{R}_{2}, \ldots, \mathscr{R}_{3}\right)$.

If $\quad \mathcal{S}\left(\mathscr{R}^{\beta}\right)=\mathcal{S}(\mathscr{R})$, that is, $\quad\left(\mu_{\mathscr{R}}^{\beta}\right)^{3}-\left(\nu_{\mathscr{R}}^{\beta}\right)^{3}=$ $\left(\mu_{\mathscr{R}}\right)^{3}-\left(\nu_{\mathscr{R}}\right)^{3}$, then by condition $\mu_{\mathscr{R}}^{\beta} \leq \mu_{\mathscr{R}}$ and $\nu_{\mathscr{R}}^{\beta} \geq \nu_{\mathscr{R}}$; thus, the accuracy function $\mathscr{A}\left(\mathscr{R}^{\beta}\right)=\left(\mu_{\mathscr{R}}^{\beta}\right)^{3}-\left(\nu_{\mathscr{R}}^{\beta}\right)^{3}=$ $\left(\mu_{\mathscr{R}}\right)^{3}-\left(\nu_{\mathscr{R}}\right)^{3}=\mathscr{A}(\mathscr{R})$. Thus,
$\operatorname{FFEWA}\left(\mathscr{R}_{1}, \mathscr{R}_{2}, \ldots, \mathscr{R}_{3}\right)=\operatorname{FFWA}\left(\mathscr{R}_{1}, \mathscr{R}_{2}, \ldots, \mathscr{R}_{\jmath}\right)$.

Hence,

$\operatorname{FFEWA}\left(\mathscr{R}_{1}, \mathscr{R}_{2}, \ldots, \mathscr{R}_{\jmath}\right) \leq \operatorname{FFWA}\left(\mathscr{R}_{1}, \mathscr{R}_{2}, \ldots, \mathscr{R}_{3}\right)$,

equality holds iff $\mathscr{R}_{1}=\mathscr{R}_{2}=\cdots=\mathscr{R}_{3}$.

Example 1 . Let $\mathscr{R}_{1}=(0.8,0.5), \quad \mathscr{R}_{2}=(0.9,0.4)$, $\mathscr{R}_{3}=(0.6,0.7)$, and $\mathscr{R}_{4}=(0.8,0.7)$ be four FFNs and $w=(0.4,0.2,0.2,0.2)^{T}$; then,

$$
\begin{aligned}
\operatorname{FFEWA}\left(\mathscr{R}_{1}, \mathscr{R}_{2}, \mathscr{R}_{3}, \mathscr{R}_{4}\right) & =\left\langle\sqrt[3]{\frac{\prod_{j=1}^{4}\left(1+\mu_{j}^{3}\right)^{w_{j}}-\prod_{j=1}^{4}\left(1-\mu_{j}^{3}\right)^{w_{j}}}{\prod_{j=1}^{4}\left(1+\mu_{j}^{3}\right)^{w_{j}}+\prod_{j=1}^{4}\left(1-\mu_{j}^{3}\right)^{w_{j}}}}, \frac{\sqrt[3]{2} \prod_{j=1}^{4} v_{j}^{w_{j}}}{\sqrt[3]{\prod_{j=1}^{4}\left(2-v_{j}^{3}\right)^{w_{j}}+\prod_{j=1}^{4}\left(v_{j}^{3}\right)^{w_{j}}}}\right\rangle \\
& =\left\langle\sqrt[3]{\frac{1.49-0.48}{1.49+0.48}}, \frac{\sqrt[3]{2} \times 0.55}{\sqrt[3]{1.80+0.16}}\right\rangle=\langle 0.80,0.55\rangle .
\end{aligned}
$$

Now,

$\operatorname{FFWA}\left(\mathscr{R}_{1}, \mathscr{R}_{2}, \mathscr{R}_{3}, \mathscr{R}_{4}\right)$

$$
=\left\langle\sqrt[3]{\left.1-\prod_{j=1}^{4}\left(1-\mu_{j}^{3}\right)^{w_{j}}, \prod_{j=1}^{4}\left(v_{j}\right)^{w_{j}}\right\rangle=\langle 0.80,0.55\rangle, \Rightarrow \operatorname{FFEWA}\left(\mathscr{R}_{1}, \mathscr{R}_{2}, \mathscr{R}_{3}, \mathscr{R}_{4}\right) \leq \operatorname{FFWA}\left(\mathscr{R}_{1}, \mathscr{R}_{2}, \mathscr{R}_{3}, \mathscr{R}_{4}\right) .}\right.
$$

Proposition 1. Let $\mathscr{R}_{j}=\left\langle\mu_{j}, v_{j}\right\rangle$ be FFNs and $w_{j}$ be the $W V$ of $\mathscr{R}_{j}$, such that $w_{j} \in[0,1]$ and $\sum_{j=1}^{j} w_{j}=1$.

(i) Idempotency: if $\mathscr{R}_{j}=\mathscr{R}_{o}=\left\langle\mu_{o}, v_{o}\right\rangle$ for all $\dot{j}$, then

$$
\operatorname{FFEWA}\left(\mathscr{R}_{1}, \mathscr{R}_{2}, \ldots, \mathscr{R}_{3}\right)=\mathscr{R}_{0} \text {. }
$$

(ii) Boundedness: let $\mathscr{R}^{-}=\left(\min _{j}\left(\mu_{j}\right), \max _{j}\left(\nu_{j}\right)\right)$ and $\mathscr{R}^{+}=\left(\max _{j}\left(\mu_{j}\right), \min _{j}\left(\nu_{j}\right)\right)$; then,

$$
\mathscr{R}^{-} \leq \operatorname{FFEWA}\left(\mathscr{R}_{1}, \mathscr{R}_{2}, \ldots, \mathscr{R}_{\jmath}\right) \leq \mathscr{R}^{+}
$$

(iii) Monotonicity: when $\mathscr{R}_{j} \leq \mathscr{P}_{j}, \forall j$, then

$\operatorname{FFEWA}\left(\mathscr{R}_{1}, \mathscr{R}_{2}, \ldots, \mathscr{R}_{s}\right) \leq \operatorname{FFEWA}\left(\mathscr{P}_{1}, \mathscr{P}_{2}, \ldots, \mathscr{P}_{s}\right)$.

Proof. (i) As $\mathscr{R}_{j}=\left\langle\mu_{o}, v_{o}\right\rangle$ are FFNs, $\forall j$, then

$$
\begin{aligned}
& \operatorname{FFEWA}\left(\mathscr{R}_{1}, \mathscr{R}_{2}, \ldots, \mathscr{R}_{j}\right)=\left\langle\sqrt[3]{\frac{\prod_{j=1}^{s}\left(1+\mu_{o}^{3}\right)^{w_{j}}-\prod_{j=1}^{j}\left(1-\mu_{o}^{3}\right)^{w_{j}}}{\prod_{j=1}^{j}\left(1+\mu_{o}^{3}\right)^{w_{j}}+\prod_{j=1}^{j}\left(1-\mu_{o}^{3}\right)^{w_{j}}}}, \frac{\sqrt[3]{2} \prod_{j=1}^{j} v_{o}^{w_{j}}}{\sqrt[\prod_{j=1}^{j}\left(2-v_{o}^{3}\right)^{w_{j}}+\prod_{j=1}^{j}\left(v_{o}^{3}\right)^{w_{j}}]{\prod_{j}}}\right\rangle
\end{aligned}
$$

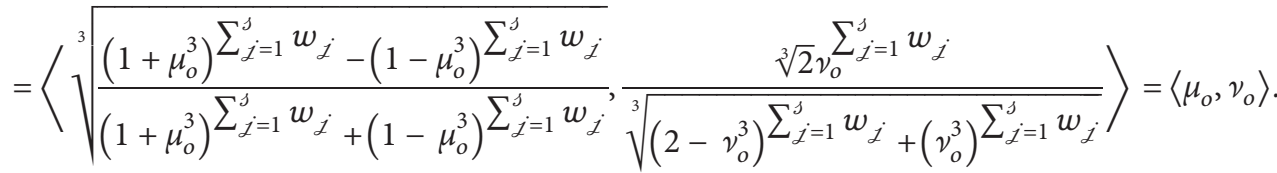


(ii) Consider $f(x)=((1-x) /(1+x)), x \in[0,1]$, then $f^{\prime}(x)=-\left(2 /(1+x)^{2}\right)<0$, so $f(x)$ is a decreasing function (DF). As $\mu_{j, \min }^{3} \leq \mu_{j}^{3} \leq \mu_{j, \text { max }}^{3}, \forall j=1,2, \ldots$, , , then $f\left(\mu_{j, \text { max }}^{3}\right) \leq f\left(\mu_{j}^{3}\right) \leq f\left(\mu_{j}^{3}\right.$, min $), \forall \dot{j}$, that is, $\left(\left(1-\mu_{j, \text { max }}^{3}\right) /\right.$ $\left.\left(1+\mu_{j, \max }^{3}\right)\right) \leq\left(\left(1-\mu_{j}^{3}\right) / \quad\left(1+\mu_{j}^{3}\right)\right) \leq\left(\left(1-\mu_{j, \min }^{3}\right) /(1+\right.$ $\left.\left.\mu_{j, \text { min }}^{3}\right)\right)$, for all $j_{j}$. Let $w_{j} \in[0,1]$ and $\sum_{j=1}^{j} w_{j}=1$, and we have

$$
\begin{aligned}
& \left(\frac{1-\mu_{j, \max }^{3}}{1+\mu_{j, \max }^{3}}\right)^{w_{j}} \leq\left(\frac{1-\mu_{j}^{3}}{1+\mu_{j}^{3}}\right)^{w_{j}} \leq\left(\frac{1-\mu_{j, \min }^{3}}{1+\mu_{j, \min }^{3}}\right)^{w_{j}} \\
& \prod_{j=1}^{j}\left(\frac{1-\mu_{j, \max }^{3}}{1+\mu_{j, \max }^{3}}\right)^{w_{j}} \leq \prod_{j=1}^{j}\left(\frac{1-\mu_{j}^{3}}{1+\mu_{j}^{3}}\right)^{w_{j}} \leq \prod_{j=1}^{j}\left(\frac{1-\mu_{j, \min }^{3}}{1+\mu_{j, \min }^{3}}\right)^{w_{j}} \\
& \Longleftrightarrow\left(\frac{1-\mu_{j, \max }^{3}}{1+\mu_{j, \max }^{3}}\right)^{\sum_{j=1}^{j} w_{j}} \leq \prod_{j=1}^{j}\left(\frac{1-\mu_{j}^{3}}{1+\mu_{j}^{3}}\right)^{w_{j}} \leq\left(\frac{1-\mu_{j, \min }^{3}}{1+\mu_{j, \min }^{3}}\right)^{\sum_{j=1}^{j} w_{j}} \\
& \Longleftrightarrow\left(\frac{1-\mu_{j, \max }^{3}}{1+\mu_{j, \max }^{3}}\right) \leq \prod_{j=1}^{3}\left(\frac{1-\mu_{j}^{3}}{1+\mu_{j}^{3}}\right)^{w_{j}} \leq\left(\frac{1-\mu_{j, \min }^{3}}{1+\mu_{j, \min }^{3}}\right) \\
& \Longleftrightarrow\left(\frac{2}{1+\mu_{j, \max }^{3}}\right) \leq 1+\prod_{j=1}^{3}\left(\frac{1-\mu_{j}^{3}}{1+\mu_{j}^{3}}\right)^{w_{j}} \leq\left(\frac{2}{1+\mu_{j, \min }^{3}}\right) \\
& \Longleftrightarrow\left(\frac{1+\mu_{j, \min }^{3}}{2}\right) \leq \frac{1}{1+\prod_{j=1}^{3}\left(\left(1-\mu_{j}^{3}\right) /\left(1+\mu_{j}^{3}\right)\right)^{w_{j}}} \leq\left(\frac{1+\mu_{j, \max }^{3}}{2}\right) \\
& \Longleftrightarrow\left(1+\mu_{j, \min }^{3}\right) \leq \frac{2}{1+\prod_{j=1}^{s}\left(\left(1-\mu_{j}^{3}\right) /\left(1+\mu_{j}^{3}\right)\right)^{w_{j}}} \leq\left(1+\mu_{j, \max }^{3}\right) \\
& \Longleftrightarrow \mu_{j, \min }^{3} \leq \frac{2}{1+\prod_{j=1}^{3}\left(\left(1-\mu_{j}^{3}\right) /\left(1+\mu_{j}^{3}\right)\right)^{w_{j}}}-1 \leq \mu_{j, \max }^{3} \\
& \Longleftrightarrow \mu_{j, \min }^{3} \leq \frac{\prod_{j=1}^{s}\left(1+\mu_{j}^{3}\right)^{w_{j}}-\prod_{j=1}^{j}\left(1-\mu_{j}^{3}\right)^{w_{j}}}{\prod_{j=1}^{s}\left(1+\mu_{j}^{3}\right)^{w_{j}}+\prod_{j=1}^{j}\left(1-\mu_{j}^{3}\right)^{w_{j}}} \leq \mu_{j, \max }^{3} .
\end{aligned}
$$

Thus,

$\mu_{j, \min } \leq \sqrt[3]{\frac{\prod_{j=1}^{s}\left(1+\mu_{j}^{3}\right)^{w_{j}}-\prod_{j=1}^{s}\left(1-\mu_{j}^{3}\right)^{w_{j}}}{\prod_{j=1}^{s}\left(1+\mu_{j}^{3}\right)^{w_{j}}+\prod_{j=1}^{s}\left(1-\mu_{j}^{3}\right)^{w_{j}}}} \leq \mu_{j, \max }$.
Consider $g(y)=((2-y) / y), y \in(0,1]$, then $g^{\prime}(y)=-$ $\left(2 / y^{2}\right)$, i.e., $g(y)$ is a DF on $(0,1]$. Since $v_{j}^{3}$, min $\leq v_{j}^{3} \leq v_{j}^{3}$, max $\forall \dot{j}$, then $g\left(v_{j, \text { max }}^{3}\right) \leq g\left(v_{j}^{3}\right) \leq g\left(v_{j, \text { min }}^{3}\right), \forall \dot{j}$, that is, $((2-$ $\left.\left.v_{j, \text { max }}^{3}\right) / v_{j, \text { max }}^{3}\right) \leq\left(\left(2-v_{j}^{3}\right) / v_{j}^{3}\right) \leq\left(\left(2-v_{j, \text { min }}^{3}\right) / v_{j, \text { min }}^{3}\right)$. Then, 


$$
\begin{aligned}
& \left(\frac{2-v_{j, \max }^{3}}{v_{j, \max }^{3}}\right)^{w_{j}} \leq\left(\frac{2-v_{j}^{3}}{v_{j}^{3}}\right)^{w_{j}} \leq\left(\frac{2-v_{j, \min }^{3}}{v_{j, \min }^{3}}\right)^{w_{j}} \\
& \prod_{j=1}^{s}\left(\frac{2-v_{j, \max }^{3}}{v_{j, \max }^{3}}\right)^{w_{j}} \leq \prod_{j=1}^{s}\left(\frac{2-v_{j}^{3}}{v_{j}^{3}}\right)^{w_{j}} \leq \prod_{j=1}^{s}\left(\frac{2-v_{j, \min }^{3}}{v_{j, \min }^{3}}\right)^{w_{j}} \\
& \Rightarrow\left(\frac{2-v_{j, \max }^{3}}{v_{j, \max }^{3}}\right)^{\sum_{j=1}^{j} w_{j}} \leq \prod_{j=1}^{j}\left(\frac{2-v_{j}^{3}}{v_{j}^{3}}\right)^{w_{j}} \leq\left(\frac{2-v_{j, \min }^{3}}{v_{j, \min }^{3}}\right)^{\sum_{j=1}^{j} w_{j}} \\
& \Rightarrow\left(\frac{2-v_{j, \max }^{3}}{v_{j, \max }^{3}}\right) \leq \prod_{j=1}^{3}\left(\frac{2-v_{j}^{3}}{v_{j}^{3}}\right)^{w_{j}} \leq\left(\frac{2-v_{j, \min }^{3}}{v_{j, \min }^{3}}\right) \\
& \Rightarrow\left(\frac{2}{v_{j, \max }^{3}}\right) \leq 1+\prod_{j=1}^{s}\left(\frac{2-v_{j}^{3}}{v_{j}^{3}}\right)^{w_{j}} \leq\left(\frac{2}{v_{j, \min }^{3}}\right) \\
& \Rightarrow\left(\frac{v_{j, \min }^{3}}{2}\right) \leq \frac{1}{1+\prod_{j=1}^{3}\left(\left(2-v_{j}^{3}\right) / v_{j}^{3}\right)^{w_{j}}} \leq\left(\frac{v_{j, \max }^{3}}{2}\right) \\
& \Rightarrow\left(v_{j, \min }^{3}\right) \leq \frac{2}{1+\prod_{j=1}^{j}\left(\left(2-v_{j}^{3}\right) / v_{j}^{3}\right)^{w_{j}}} \leq\left(v_{j, \max }^{3}\right) \\
& \Rightarrow v_{j, \min }^{3} \leq \frac{2}{1+\prod_{j=1}^{j}\left(\left(2-v_{j}^{3}\right) / v_{j}^{3}\right)^{w_{j}}} \leq v_{j, \max }^{3} \\
& \Rightarrow v_{j, \min }^{3} \leq \frac{2 \prod_{j=1}^{3}\left(v_{j}^{3}\right)^{w_{j}}}{\prod_{j=1}^{3}\left(2-v_{j}^{3}\right)^{w_{j}}+\prod_{j=1}^{s}\left(v_{j}^{3}\right)^{w_{j}}} \leq v_{j, \max }^{3} \\
& \Rightarrow v_{j, \min } \leq \frac{\sqrt[3]{2} \prod_{j=1}^{j} v_{j}^{w_{j}}}{\sqrt[3]{\prod_{j=1}^{j}\left(2-v_{j}^{3}\right)^{w_{j}}+\prod_{j=1}^{j}\left(v_{j}^{3}\right)^{w_{j}}}} \leq v_{j, \max } .
\end{aligned}
$$

Let FFEWA $\left(\mathscr{R}_{1}, \mathscr{R}_{2}, \ldots, \mathscr{R}_{3}\right)=\mathscr{R}=\left\langle\mu_{\mathscr{R}}, v_{\mathscr{R}}\right\rangle$; then, from equations (46) and (47),

$$
\begin{aligned}
& \mu_{\text {min }} \leq \mu_{\mathscr{R}} \leq \mu_{\text {max }}, \\
& \nu_{\text {min }} \leq \nu_{\mathscr{R}} \leq v_{\text {max }},
\end{aligned}
$$

where $\mu_{\min }=\min _{j}\left\{\mu_{j}\right\}, \quad \mu_{\max }=\max _{j}\left\{\mu_{j}\right\}, \quad v_{\min }=\min _{j}$ $\left\{v_{j}\right\}$, and $v_{\max }=\max _{j}\left\{v_{j}\right\}$. So, $\delta(\mathscr{R})=\mu_{\mathscr{R}}^{3}-v_{\mathscr{R}}^{3} \leq \mu_{\max }^{3}-$ $v_{\min }^{3}=\mathcal{S}\left(\mathscr{R}^{+}\right) \quad$ and $\quad \mathcal{S}(\mathscr{R})=\mu_{\mathscr{R}}^{3}-v_{\mathscr{R}}^{3} \geq \mu_{\min }^{3}-$ $\nu_{\text {max }}^{3}=\mathcal{S}\left(\mathscr{R}^{-}\right)$. As $S(\mathscr{R})<S\left(\mathscr{R}^{+}\right)$and $S(\mathscr{R})>S\left(\mathscr{R}^{-}\right)$, so

$$
\mathscr{R}^{-} \leq \operatorname{FFEWA}\left(\mathscr{R}_{1}, \mathscr{R}_{2}, \ldots, \mathscr{R}_{3}\right) \leq \mathscr{R}^{+} .
$$

(iii) It is similar to (ii), so we omit it.

\section{Fermatean Fuzzy Einstein Ordered Weighted Averaging Operators}

Definition 8. Let $\mathscr{R}_{j}=\left\langle\mu_{j}, v_{j}\right\rangle$ be a family of FFNs and $w_{j}$ be the WV of $\mathscr{R}_{j}$ with $w_{j}>0$ and $\sum_{j=1}^{j} w_{j}=1$; then, FFEOWA operator is a mapping $\mathbb{Q}^{j} \longrightarrow \mathbb{Q}$ such that

$$
\begin{aligned}
& \operatorname{FFEOWA}\left(\mathscr{R}_{1}, \mathscr{R}_{2}, \ldots, \mathscr{R}_{\jmath}\right) \\
& =w_{1} \cdot{ }_{\varepsilon} \mathscr{R}_{\varrho(1)} \oplus_{\varepsilon} w_{2} \cdot{ }_{\varepsilon} \mathscr{R}_{\varrho(2)} \oplus_{\varepsilon} \cdots \oplus_{\varepsilon} w_{\jmath} \cdot{ }_{\varepsilon} \mathscr{R}_{\varrho(\jmath)},
\end{aligned}
$$

where $(\varrho(1), \varrho(2), \ldots, \varrho(\jmath))$ is the permutation of $(j=1,2, \ldots, j)$ such that $\mathscr{R}_{\varrho(j-1)} \geq \mathscr{R}_{\varrho(j)}, \forall j=$ $1,2, \ldots, 3$.

Theorem 7. Let $\mathscr{R}_{j}=\left(\mu_{j}, v_{j}\right)$ be FFNs; then, the aggregated value by using FFEOWA is an FFN and

$$
\operatorname{FFEOWA}\left(\mathscr{R}_{1}, \mathscr{R}_{2}, \ldots, \mathscr{R}_{3}\right)
$$

$$
\begin{gathered}
=\left\langle\sqrt[3]{\frac{\prod_{j=1}^{s}\left(1+\mu_{\varrho(j)}^{3}\right)^{w_{j}}-\prod_{j=1}^{j}\left(1-\mu_{\varrho(j)}^{3}\right)^{w_{j}}}{\prod_{j=1}^{j}\left(1+\mu_{\varrho(j)}^{3}\right)^{w_{j}}+\prod_{j=1}^{j}\left(1-\mu_{\varrho(j)}^{3}\right)^{w_{j}}}},\right. \\
\left.\frac{\sqrt[3]{2} \prod_{j=1}^{j} v_{\varrho(j)}^{w_{j}}}{\prod_{j=1}^{j}\left(2-v_{\varrho(j)}^{3}\right)^{w_{j}}+\prod_{j=1}^{j}\left(v_{\varrho(j)}^{3}\right)^{w_{j}}}\right\rangle .
\end{gathered}
$$


Proof. It is similar to Theorem 4.

We give some properties without their proofs.

Corollary 2. The FFEOWA and FFOWA operators have the relation:

$\operatorname{FFEOWA}\left(\mathscr{R}_{1}, \mathscr{R}_{2}, \ldots, \mathscr{R}_{3}\right) \leq \operatorname{FFOWA}\left(\mathscr{R}_{1}, \mathscr{R}_{2}, \ldots, \mathscr{R}_{3}\right)$.

Example 2. Let $\mathscr{R}_{1}=(0.6,0.7), \quad \mathscr{R}_{2}=(0.8,0.7)$, $\mathscr{R}_{3}=(0.6,0.9)$, and $\mathscr{R}_{4}=(0.9,0.4)$ be four FFNs and $w=$ $(0.3,0.3,0.2,0.2)^{T}$ as

$$
\begin{aligned}
& \mathcal{S}\left(\mathscr{R}_{1}\right)=(0.6)^{3}-(0.7)^{3}=-0.13, \\
& \mathcal{S}\left(\mathscr{R}_{2}\right)=(0.8)^{3}-(0.7)^{3}=0.17, \\
& \mathcal{S}\left(\mathscr{R}_{3}\right)=(0.6)^{3}-(0.9)^{3}=-0.51, \\
& \mathcal{S}\left(\mathscr{R}_{4}\right)=(0.9)^{3}-(0.4)^{3}=0.67 .
\end{aligned}
$$

Since $\mathcal{S}\left(\mathscr{R}_{4}\right)>\mathcal{S}\left(\mathscr{R}_{2}\right)>\mathcal{S}\left(\mathscr{R}_{1}\right)>\mathcal{S}\left(\mathscr{R}_{3}\right)$, therefore

$$
\begin{aligned}
& \mathscr{R}_{\varrho(1)}=\mathscr{R}_{4}=(0.9,0.4), \\
& \mathscr{R}_{\varrho(2)}=\mathscr{R}_{2}=(0.8,0.7), \\
& \mathscr{R}_{\varrho(3)}=\mathscr{R}_{1}=(0.6,0.7), \\
& \mathscr{R}_{\varrho(4)}=\mathscr{R}_{3}=(0.6,0.9) .
\end{aligned}
$$

Thus, by applying the FFEOWA operator, we obtain

$$
\begin{aligned}
\operatorname{FFEOWA}\left(\mathscr{R}_{1}, \mathscr{R}_{2}, \mathscr{R}_{3}, \mathscr{R}_{4}\right) & =\left\langle\sqrt[3]{\left.\frac{\prod_{j=1}^{4}\left(1+\mu_{\varrho(j)}^{3}\right)^{w_{j}}-\prod_{j=1}^{4}\left(1-\mu_{\varrho(j)}^{3}\right)^{w_{j}}}{\prod_{j=1}^{4}\left(1+\mu_{\varrho(j)}^{3}\right)^{w_{j}}+\prod_{j=1}^{4}\left(1-\mu_{\varrho(j)}^{3}\right)^{w_{j}}}, \frac{\sqrt[3]{\prod^{2}} \prod_{j=1}^{4}\left(2-v_{\varrho(j)}^{3}\right)^{w_{j}}+\prod_{j(j)}^{w_{j}}\left(\nu_{\varrho(j)}^{3}\right)^{w_{j}}}{\prod_{j=1}^{4}}\right\rangle}\right. \\
& =\left\langle\sqrt[3]{\frac{1.44-0.49}{1.44+0.49}}, \frac{\sqrt[3]{2} \times 0.66}{\sqrt[3]{1.62+0.66}}\right\rangle=\langle 0.79,0.55\rangle .
\end{aligned}
$$

Now,

$$
\operatorname{FFOWA}\left(\mathscr{R}_{1}, \mathscr{R}_{2}, \mathscr{R}_{3}, \mathscr{R}_{4}\right)
$$$$
=\left\langle\sqrt[3]{1-\prod_{j=1}^{4}\left(1-\mu_{\varrho(j)}^{3}\right)^{w_{j}}}, \prod_{j=1}^{4}\left(v_{\varrho(j)}\right)^{w_{j}}\right\rangle=\langle 0.80,0.66\rangle,
$$$$
\Rightarrow \operatorname{FFEOWA}\left(\mathscr{R}_{1}, \mathscr{R}_{2}, \mathscr{R}_{3}, \mathscr{R}_{4}\right)<\operatorname{FFOWA}\left(\mathscr{R}_{1}, \mathscr{R}_{2}, \mathscr{R}_{3}, \mathscr{R}_{4}\right) \text {. }
$$

Proposition 1. Let $\mathscr{R}_{j}=\left\langle\mu_{j}, v_{j}\right\rangle$ be FFNs and $w_{j}$ be the $W V$ of $\mathscr{R}_{j}$, such that $w_{j} \in[0,1]$ and $\sum_{j=1}^{j} w_{j}=1$.

(i) Idempotency: if $\mathscr{R}_{j}=\mathscr{R}_{o}=\left\langle\mu_{o}, v_{o}\right\rangle, \forall j$, then

$$
\operatorname{FFEOWA}\left(\mathscr{R}_{1}, \mathscr{R}_{2}, \ldots, \mathscr{R}_{3}\right)=\mathscr{R}_{o}
$$

(ii) Boundedness: let $\mathscr{R}^{-}=\left(\min _{j}\left(\mu_{j}\right), \max _{j}\left(\nu_{j}\right)\right)$ and $\mathscr{R}^{+}=\left(\max _{j}\left(\mu_{j}\right), \min _{j}\left(\nu_{j}\right)\right) ;$ then,

$$
\mathscr{R}^{-} \leq \operatorname{FFEOWA}\left(\mathscr{R}_{1}, \mathscr{R}_{2}, \ldots, \mathscr{R}_{\jmath}\right) \leq \mathscr{R}^{+}
$$

(iii) Monotonicity: when $\mathscr{R}_{j} \leq \mathscr{P}_{j}, \forall j$, then
$\operatorname{FFEOWA}\left(\mathscr{R}_{1}, \mathscr{R}_{2}, \ldots, \mathscr{R}_{3}\right) \leq \operatorname{FFEOWA}\left(\mathscr{P}_{1}, \mathscr{P}_{2}, \ldots, \mathscr{P}_{s}\right)$.

\section{Generalized Fermatean Fuzzy Einstein Weighted Averaging Operators}

Definition 9. Let $\mathscr{R}_{j}=\left\langle\mu_{j}, v_{j}\right\rangle$ be a collection of FFNs and $w_{j}$ be the WV of $\mathscr{R}_{j}$ with $w_{j}>0$ and $\sum_{j=1}^{j} w_{j}=1$; then, GFFEWA operator is a mapping $\mathbb{Q}^{j} \longrightarrow \mathscr{Q}$ such that

$$
\operatorname{GFFEWA}\left(\mathscr{R}_{1}, \mathscr{R}_{2}, \ldots, \mathscr{R}_{j}\right)=\left(\oplus_{j=1}^{s}\left(w_{j} \cdot{ }_{\varepsilon} \mathscr{R}_{j}^{\lambda}\right)\right)^{1 / \lambda} \text {, }
$$

where $\lambda>0$.

Particularly,

(i) If $\lambda=1$, then GFFEWA becomes FFEWA

(ii) If $w=((1 / s),(1 / s), \ldots,(1 / s))^{T}$, then GFFEWA $\left(\mathscr{R}_{1}, \mathscr{R}_{2}, \ldots, \mathscr{R}_{3}\right)=\left((1 / s) \cdot{ }_{\varepsilon} \oplus_{j=1}^{s} \mathscr{R}_{j}^{\lambda}\right)^{1 / \lambda}$

Theorem 8. Let $\mathscr{R}_{j}=\left\langle\mu_{j}, v_{j}\right\rangle$ be FFNs and $w_{j}$ be the WV of $\mathscr{R}_{j}$ with $w_{j}>0$ and $\sum_{j=1}^{j} w_{j}=1$; then, the aggregated value by applying the GFFEWA operator is an FFN and 
$\operatorname{GFFEWA}\left(\mathscr{R}_{1}, \mathscr{R}_{2}, \ldots, \mathscr{R}_{3}\right)$

$$
\begin{aligned}
& =\left\langle\frac{\sqrt[3]{2}\left\{\prod_{j=1}^{s}\left\{\left(2-\mu_{j}^{3}\right)^{\lambda}+3\left(\mu_{j}^{3}\right)^{\lambda}\right\}^{w_{j}}-\prod_{j=1}^{s}\left\{\left(2-\mu_{j}^{3}\right)^{\lambda}+\left(\mu_{j}^{3}\right)^{\lambda}\right\}^{w_{j}}\right\}^{1 / 3 \lambda}}{\sqrt[3]{\left(\prod_{j=1}^{s}\left\{\left(2-\mu_{j}^{3}\right)^{\lambda}+3\left(\mu_{j}^{3}\right)^{\lambda}\right\}^{w_{j}}+3 \prod_{j=1}^{s}\left\{\left(2-\mu_{j}^{3}\right)^{\lambda}-\left(\mu_{j}^{3}\right)^{\lambda}\right\}^{w_{j}}\right)^{1 / \lambda}+\left(\prod_{j=1}^{s}\left\{\left(2-\mu_{j}^{3}\right)^{\lambda}+3\left(\mu_{j}^{3}\right)^{\lambda}\right\}^{w_{j}}-\prod_{j=1}^{s}\left\{\left(2-\mu_{j}^{3}\right)^{\lambda}-\left(\mu_{j}^{3}\right)^{\lambda}\right\}^{w_{j}}\right)^{1 / \lambda}}}\right. \\
& \left.\cdot \sqrt[3]{\frac{\left(\prod_{j=1}^{s}\left\{\left(1+v_{j}^{3}\right)^{\lambda}+3\left(1-v_{j}^{3}\right)^{\lambda}\right\}^{w_{j}}+3 \prod_{j=1}^{s}\left\{\left(1+v_{j}^{3}\right)^{\lambda}-\left(1-v_{j}^{3}\right)^{\lambda}\right\}^{w_{j}}\right)^{1 / \lambda}-\left(\prod_{j=1}^{s}\left\{\left(1+v_{j}^{3}\right)^{\lambda}+3\left(1-v_{j}^{3}\right)^{\lambda}\right\}^{w_{j}}-\prod_{j=1}^{s}\left\{\left(1+v_{j}^{3}\right)^{\lambda}-\left(1-v_{j}^{3}\right)^{\lambda}\right\}^{w_{j}}\right)^{1 / \lambda}}{\left(\prod_{j=1}^{s}\left\{\left(1+v_{j}^{3}\right)^{\lambda}+3\left(1-v_{j}^{3}\right)^{\lambda}\right\}^{w_{i}}+3 \prod_{j=1}^{s}\left\{\left(1+v_{j}^{3}\right)^{\lambda}-\left(1-v_{j}^{3}\right)^{\lambda}\right\}^{w_{j}}\right)^{1 / \lambda}+\left(\prod_{j=1}^{s}\left\{\left(1+v_{j}^{3}\right)^{\lambda}+3\left(1-v_{j}^{3}\right)^{\lambda}\right\}^{w_{j}}-\prod_{j=1}^{s}\left\{\left(1+v_{j}^{3}\right)^{\lambda}-\left(1-v_{j}^{3}\right)^{\lambda}\right\}^{w_{j}}\right)^{1 / \lambda}}}\right\rangle .
\end{aligned}
$$

\section{Proof. Since}

$$
\begin{aligned}
& \mathscr{R}_{j}^{\lambda}=\left\langle\frac{\sqrt[3]{2} \mu_{j}^{\lambda}}{\sqrt[3]{\left(2-\mu_{j}^{3}\right)^{\lambda}+\left(\mu_{j}^{3}\right)^{\lambda}}}, \sqrt[3]{\frac{\left(1+v_{j}^{3}\right)^{\lambda}-\left(1-v_{j}^{3}\right)^{\lambda}}{\left(1+v_{j}^{3}\right)^{\lambda}+\left(1-v_{j}^{3}\right)^{\lambda}}}\right\rangle \\
& \Rightarrow \oplus_{j=1}^{3} w_{j} \cdot{ }_{\varepsilon} \mathscr{R}_{j}^{\lambda}=\left\langle\sqrt[3]{\frac{\prod_{j=1}^{3}\left(1+\left(2 \mu_{j}^{3 \lambda} /\left(\left(2-\mu_{j}^{3}\right)^{\lambda}+\left(\mu_{j}^{3}\right)^{\lambda}\right)\right)\right)^{w_{j}}-\prod_{j=1}^{j}\left(1-\left(2 \mu_{j}^{3 \lambda} /\left(\left(2-\mu_{j}^{3}\right)^{\lambda}+\left(\mu_{j}^{3}\right)^{\lambda}\right)\right)\right)^{w_{j}}}{\prod_{j=1}^{3}\left(1+\left(2 \mu_{j}^{3 \lambda} /\left(\left(2-\mu_{j}^{3}\right)^{\lambda}+\left(\mu_{j}^{3}\right)^{\lambda}\right)\right)\right)^{w_{j}}+\prod_{j=1}^{j}\left(1-\left(2 \mu_{j}^{3 \lambda} /\left(\left(2-\mu_{j}^{3}\right)^{\lambda}+\left(\mu_{j}^{3}\right)^{\lambda}\right)\right)\right)^{w_{j}}}},\right. \\
& \sqrt[3]{2} \prod_{j=1}^{s}\left(\sqrt[3]{\left(\left(1+v_{j}^{3}\right)^{\lambda}-\left(1-v_{j}^{3}\right)^{\lambda}\right) /\left(\left(1+v_{j}^{3}\right)^{\lambda}+\left(1-v_{j}^{3}\right)^{\lambda}\right)}\right)^{w_{j}} \\
& \left.\sqrt[3]{\prod_{j=1}^{s}\left(2-\left(\left(\left(1+v_{j}^{3}\right)^{\lambda}-\left(1-v_{j}^{3}\right)^{\lambda}\right) /\left(\left(1+v_{j}^{3}\right)^{\lambda}+\left(1-v_{j}^{3}\right)^{\lambda}\right)\right)\right)^{w_{j}}+\prod_{j=1}^{s}\left(\left(\left(1+v_{j}^{3}\right)^{\lambda}-\left(1-v_{j}^{3}\right)^{\lambda}\right) /\left(\left(1+v_{j}^{3}\right)^{\lambda}+\left(1-v_{j}^{3}\right)^{\lambda}\right)\right)^{w_{j}}}\right\rangle \\
& =\left\langle\sqrt[3]{\frac{\prod_{j=1}^{s}\left\{\left(2-\mu_{j}^{3}\right)^{\lambda}+3\left(\mu_{j}^{3}\right)^{\lambda}\right\}^{w_{j}}-\prod_{j=1}^{s}\left\{\left(2-\mu_{j}^{3}\right)^{\lambda}-\left(\mu_{j}^{3}\right)^{\lambda}\right\}^{w_{j}}}{\prod_{j=1}^{s}\left\{\left(2-\mu_{j}^{3}\right)^{\lambda}+3\left(\mu_{j}^{3}\right)^{\lambda}\right\}^{w_{j}}+\prod_{j=1}^{s}\left\{\left(2-\mu_{j}^{3}\right)^{\lambda}-\left(\mu_{j}^{3}\right)^{\lambda}\right\}^{w_{j}}}}\right. \\
& \sqrt[3]{2} \prod_{j=1}^{s}\left(\sqrt[3]{\left(1+v_{j}^{3}\right)^{\lambda}-\left(1-v_{j}^{3}\right)^{\lambda}}\right)^{w_{j}}
\end{aligned}
$$

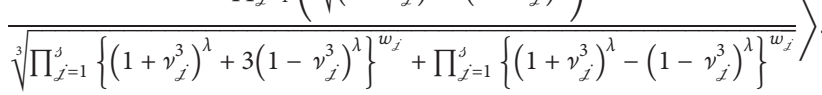

\section{Therefore,}

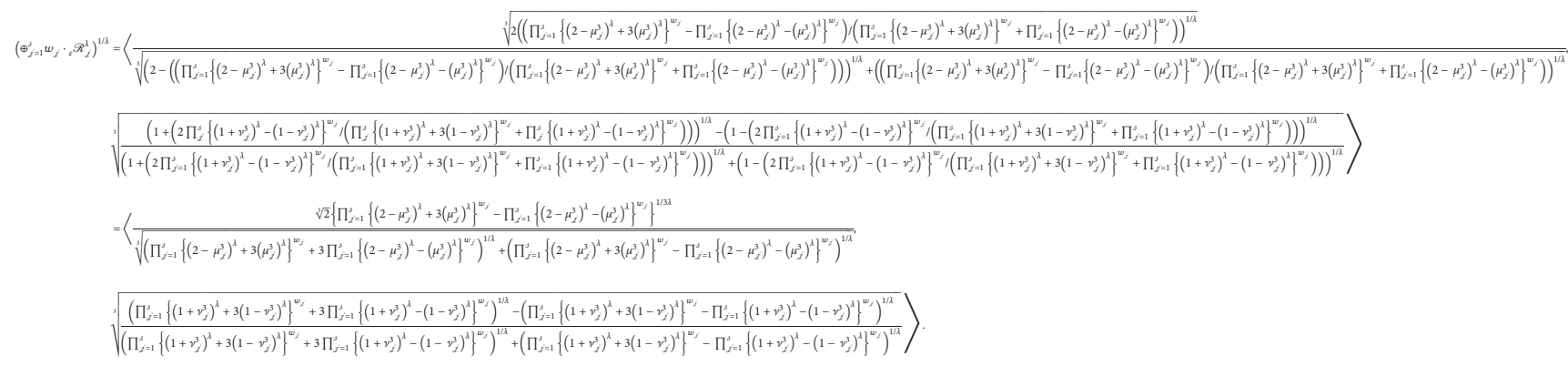


When $\lambda=1$, then

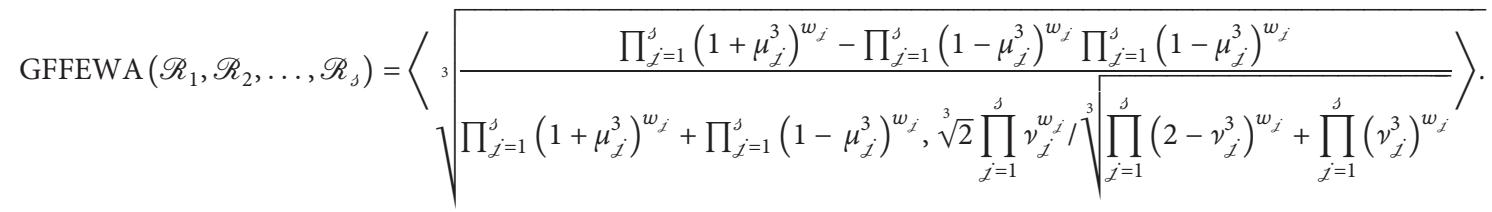

\section{Generalized Fermatean Fuzzy Einstein Ordered Weighted Averaging Operators}

Definition 10. Let $\mathscr{R}_{j}=\left\langle\mu_{j}, v_{j}\right\rangle$ be a collection of FFNs and $w_{j}$ be the WV of $\mathscr{R}_{j}$ with $w_{j}>0$ and $\sum_{j=1}^{j} w_{j}=1$; then, the GFFEOWA operator is a mapping $Q^{s} \stackrel{\mathcal{Q}}{\longrightarrow}$ such that

$$
\operatorname{GFFEOWA}\left(\mathscr{R}_{1}, \mathscr{R}_{2}, \ldots, \mathscr{R}_{\jmath}\right)=\left(\oplus_{j=1}^{\jmath}\left(w_{j} \cdot \mathscr{R}_{\varrho(j)}^{\lambda}\right)\right)^{1 / \lambda},
$$

where $\lambda>0$.

Theorem 9. Let $\mathscr{R}_{j}=\left\langle\mu_{j}, v_{j}\right\rangle$ be FFNs and $w_{j}$ be the WV of $\mathscr{R}_{j}$ with $w_{j}>0$ and $\sum_{j=1}^{j} w_{j}=1$; then, the aggregated value by applying the GFFEOWA operator is an FFN and

$$
\begin{aligned}
& \operatorname{GFFEOWA}\left(\mathscr{R}_{1}, \mathscr{R}_{2}, \ldots, \mathscr{R}_{j}\right)=\left\langle\frac{\sqrt[3]{2}\left\{\prod_{j=1}^{s}\left\{\left(2-\mu_{\mathrm{e}(j)}^{3}\right)^{\lambda}+3\left(\mu_{\mathrm{e}(j)}^{3}\right)^{\lambda}\right\}^{w_{j}}-\prod_{j=1}^{s}\left\{\left(2-\mu_{\mathrm{e}(j)}^{3}\right)^{\lambda}-\left(\mu_{\mathrm{e}(j)}^{3}\right)^{\lambda}\right\}^{w_{j}}\right\}^{1 / 3 \lambda}}{\sqrt{\left(\prod_{j=1}^{s}\left\{\left(2-\mu_{\mathrm{e}(j)}^{3}\right)^{\lambda}+3\left(\mu_{\mathrm{e}(j)}^{3}\right)^{\lambda}\right\}^{w_{j}}+3 \prod_{j=1}^{s}\left\{\left(2-\mu_{\mathrm{e}(j)}^{3}\right)^{\lambda}-\left(\mu_{\mathrm{e}(j)}^{3}\right)^{\lambda}\right\}^{w_{j}}\right)^{1 / \lambda}+\left(\prod_{j=1}^{s}\left\{\left(2-\mu_{\mathrm{e}(j)}^{3}\right)^{\lambda}+3\left(\mu_{\mathrm{e}(j)}^{3}\right)^{\lambda}\right\}^{w_{j}}-\prod_{j=1}^{j}\left\{\left(2-\mu_{\mathrm{e}(j)}^{3}\right)^{\lambda}-\left(\mu_{\mathrm{e}(j)}^{3}\right)^{\lambda}\right\}^{w_{j}}\right)^{1 / \lambda}}}\right.
\end{aligned}
$$

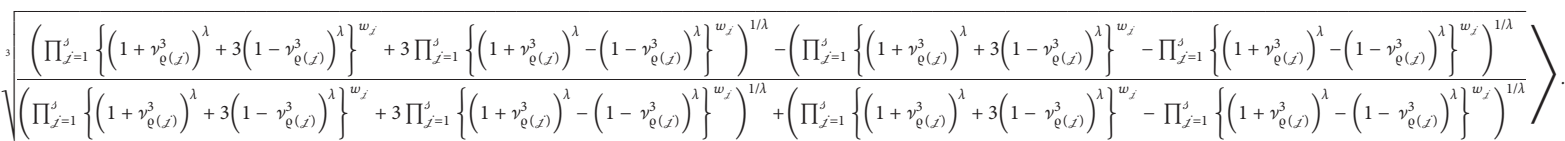

Proof. It is similar to Theorem 6, and we can prove it.

When $\lambda=1$, then

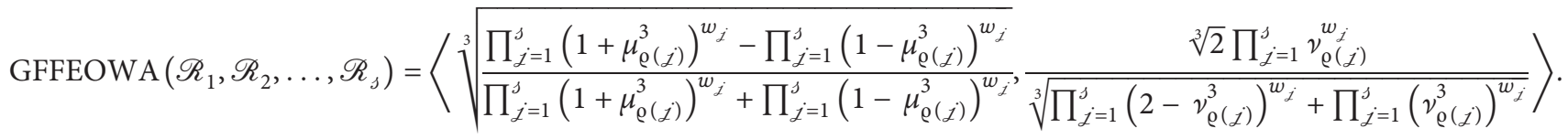

\section{MADM Problem Using FF Information}

To handle an MADM problem under FF environment, let $\mathscr{K}=\left\{\mathscr{K}_{1}, \mathscr{K}_{2}, \ldots, \mathscr{K}_{m}\right\}$ be a set of possible alternatives and $\mathscr{J}=\left\{\mathscr{J}_{1}, \mathscr{J}_{2}, \ldots, \mathscr{J}_{3}\right\}$ be a set of possible attributes chosen by the decision maker. Let $w=\left(w_{1}, w_{2}, \ldots, w_{\jmath}\right)^{T}$ be the WV with $w_{j}>0$ and $\sum_{j=1}^{j} w_{j}=1$. Suppose that $\widetilde{\mathscr{E}}=\left(\mu_{l_{j}}, v_{l_{j}}\right)_{m \times s}$ is the FF decision matrix (FFDM), where $\mu_{l_{j}}$ and $\nu_{l_{j}}$ are the MD and NMD of the alternative $\mathscr{K}_{l}$ for the attribute $\mathscr{J}_{j}$, respectively, where $0 \leq \mu_{l_{j}}^{3}+v_{l_{j}}^{3} \leq 1$.

The following Algorithm 1 is used to solve the MADM problem with FFN based on using the GFFEWA operator. 
(1) Input: selection of suitable alternatives and attributes.

(2) Use the FFDM and GFFEWA operator:

Algorithm 1

$\mathscr{B}_{l}=\operatorname{GFFEWA}\left(\mathscr{K}_{l 1}, \mathscr{K}_{l 2}, \ldots, \mathscr{K}_{l s}\right)$

$=\left\langle\frac{\sqrt[3]{2}\left\{\prod_{j^{\prime}=1}^{s}\left\{\left(2-\mu_{l_{j}}^{3}\right)^{\lambda}+3\left(\mu_{l_{j}}^{3}\right)^{\lambda}\right\}^{w_{j}}-\prod_{j^{\prime}=1}^{s}\left\{\left(2-\mu_{l_{j}}^{3}\right)^{\lambda}-\left(\mu_{l_{j}}^{3}\right)^{\lambda}\right\}^{w_{j}}\right\}^{1 / 3 \lambda}}{\sqrt[3]{\left(\prod_{j=1}^{s}\left\{\left(2-\mu_{l_{j}}^{3}\right)^{\lambda}+3\left(\mu_{l_{j}}^{3}\right)^{\lambda}\right\}^{w_{j}}+3 \prod_{j=1}^{s}\left\{\left(2-\mu_{l_{j}}^{3}\right)^{\lambda}-\left(\mu_{l_{j}}^{3}\right)^{\lambda}\right\}^{w_{j}}\right)^{1 / \lambda}+\left(\prod_{j=1}^{s}\left\{\left(2-\mu_{l_{j}}^{3}\right)^{\lambda}+3\left(\mu_{l_{j}}^{3}\right)^{\lambda}\right\}^{w_{j}}-\prod_{j^{\prime}=1}^{s}\left\{\left(2-\mu_{l_{j}}^{3}\right)^{\lambda}-\left(\mu_{l_{j}}^{3}\right)^{\lambda}\right\}^{w_{j}}\right)^{1 / \lambda}}}\right.$

$\sqrt[3]{\frac{\left(\prod_{j^{\prime}=1}^{s}\left\{\left(1+v_{l_{j}}^{3}\right)^{\lambda}+3\left(1-v_{l_{j}}^{3}\right)^{\lambda}\right\}^{w_{j}}+3 \prod_{j^{\prime}=1}^{s}\left\{\left(1+v_{l_{j}}^{3}\right)^{\lambda}-\left(1-v_{l_{j}}^{3}\right)^{\lambda}\right\}^{w_{j}}\right)^{1 / \lambda}-\left(\prod_{j=1}^{s}\left\{\left(1+v_{l_{j}}^{3}\right)^{\lambda}+3\left(1-v_{l_{j}}^{3}\right)^{\lambda}\right\}^{w_{j}}-\prod_{j^{\prime}=1}^{s}\left\{\left(1+v_{l_{j}}^{3}\right)^{\lambda}-\left(1-v_{l_{j}}^{3}\right)^{\lambda}\right\}^{w_{j}}\right)^{1 / \lambda}}{\left(\prod_{j=1}^{s}\left\{\left(1+v_{l_{j}}^{3}\right)^{\lambda}+3\left(1-v_{l_{j}}^{3}\right)^{\lambda}\right\}^{w_{j}}+3 \prod_{j^{\prime}=1}^{s}\left\{\left(1+v_{l_{j}}^{3}\right)^{\lambda}-\left(1-v_{l_{j}}^{3}\right)^{\lambda}\right\}^{w_{j}}\right)^{1 / \lambda}+\left(\prod_{j=1}^{s}\left\{\left(1+v_{l_{j}}^{3}\right)^{\lambda}+3\left(1-v_{l_{j}}^{3}\right)^{\lambda}\right\}^{w_{j}}-\prod_{j^{\prime}=1}^{s}\left\{\left(1+v_{l_{j}}^{3}\right)^{\lambda}-\left(1-v_{l_{j}}^{3}\right)^{\lambda}\right\}^{w_{j}}\right)^{1 / \lambda}}}$,

for overall preference values $\mathscr{B}_{l}(l=1,2, \ldots, m)$ of the alternatives $\mathscr{K}_{l}$.

(3) Use the score function $\mathcal{S}\left(\mathscr{B}_{l}\right)(l=1,2, \ldots, m)$ for the ranking of alternatives. If score values are equal, then compute the accuracy functions $\mathscr{A}\left(\mathscr{B}_{l}\right)$ and rank according to these values.

Output: the alternative containing maximum score value will be the decision.

8.1. Selection of an Effective Sanitizer to Reduce Coronavirus. Hand sanitizer is a liquid or gel mostly used to reduce infectious agents on the hands. Alcohol-based hand sanitizers are preferred for hand washing in most healthcare settings. The Centers for Disease Control and Prevention (CDC) advise the people to wash hands with soap and water to restrain the spread of infections and decrease the endanger of getting sick. In shortage of soap and water, CDC suggests people to use an alcohol-based (at least 60 percent) hand sanitizer. According to the World Health Organization (WHO), in this pandemic situation of coronavirus, good hygiene and physical distancing are the best ways to protect ourself and everyone around us from coronavirus. This virus spreads by a person who has the disease and also spread by touching a sick person. We cannot isolate ourselves entirely to prudent from coronavirus. So, good hand hygiene can be the final barrier between us and the disease. WHO recommends alcohol-based hand sanitizers to remove the novel coronavirus. Alcohol-based hand sanitizer works to prevent the proteins of microbes-including bacteria and some viruses-from functioning normally. Hand sanitizers must contain ethanol, isopropanol, n-propanol, or a combination of these alcohols. All are effective against viruses such as the novel coronavirus.

Demand of a hand sanitizer is increased in such critical situation of COVID-19. Due to increasing demand, it is difficult to get good and effective hand sanitizers in local markets. Increasing demand has also led to low quality hand sanitizers entering the market. The main motive of this application is to select an effective sanitizer to mitigate transmission of coronavirus by applying the GFFYWA operator. Let $\mathscr{K}=\left\{\mathscr{K}_{1}, \mathscr{K}_{2}, K_{3}, \mathscr{K}_{4}\right\}$ be a set of sanitizers. Let $\mathscr{J}=\left\{\mathscr{J}_{1}, \mathscr{J}_{2}, \mathscr{J}_{3}\right\}$ be a set of three attributes for the evaluation of an effective sanitizer, where

$$
\begin{aligned}
& \mathscr{J}_{1}: \text { represents quantity of ethanol, } \\
& \mathscr{J}_{2}: \text { represents quantity of glycerol, }
\end{aligned}
$$

$\mathscr{F}_{3}$ : represents quantity of hydrogenperoxide.

(1) The FFDM is shown in Table 2.

(2) The weights assigned by the decision maker are

$$
\begin{aligned}
w_{1} & =0.60, \\
w_{2} & =0.25, \\
w_{3} & =0.15, \\
\sum_{j=1}^{3} w_{j} & =1 .
\end{aligned}
$$

We use the GFFEWA operator for the selection of an effective sanitizer.

Step 1. For performance values $\mathscr{B}_{l}$ of sanitizers, use the GFFEWA operator for $\lambda=1$ :

$$
\begin{aligned}
& \mathscr{B}_{1}=(0.64,0.48), \\
& \mathscr{B}_{2}=(0.46,0.28), \\
& \mathscr{B}_{3}=(0.72,0.31), \\
& \mathscr{B}_{4}=(0.77,0.34) .
\end{aligned}
$$

Step 2. Calculate the scores $\mathcal{S}\left(\mathscr{B}_{l}\right)$ of FFNs $\mathscr{B}_{l}$ and rank the sanitizers: 
TABLE 2: FFDM.

\begin{tabular}{lccc}
\hline$\widetilde{\mathscr{E}}$ & $\mathscr{J}_{1}$ & $\mathscr{J}_{2}$ & $\mathscr{J}_{3}$ \\
\hline $\mathscr{K}_{1}$ & $(0.6,0.7)$ & $(0.6,0.2)$ & $(0.8,0.4)$ \\
$\mathscr{K}_{2}$ & $(0.4,0.2)$ & $(0.6,0.4)$ & $(0.3,0.6)$ \\
$\mathscr{K}_{3}$ & $(0.8,0.3)$ & $(0.5,0.3)$ & $(0.6,0.4)$ \\
$\mathscr{K}_{4}$ & $(0.7,0.5)$ & $(0.8,0.1)$ & $(0.9,0.6)$ \\
\hline
\end{tabular}

$$
\begin{aligned}
& \mathcal{S}\left(\mathscr{B}_{1}\right)=0.15, \\
& \mathcal{S}\left(\mathscr{B}_{2}\right)=0.08, \\
& \mathcal{S}\left(\mathscr{B}_{3}\right)=0.34, \\
& \mathcal{S}\left(\mathscr{B}_{4}\right)=0.42 .
\end{aligned}
$$

The ranking of sanitizers is

$$
\mathscr{K}_{4}>\mathscr{K}_{3}>\mathscr{K}_{1}>\mathscr{K}_{2} .
$$

Step 3. Therefore, $\mathscr{K}_{4}$ is the best sanitizer.

\section{Validity Test}

For the validity and authenticity of MADM methods, Wang and Triantaphyllou [53] developed testing criteria, given as follows:

(i) Criterion 1: a MADM technique is valid if the most desirable alternative remains same on changes a nonoptimal alternative with some other poor or weak alternative, without changing the respective decision criteria

(ii) Criterion 2: the transitive property should be followed by a valid MADM technique

(iii) Criterion 3: the ranking result of alternatives should not change on splitting the problem into the smaller subproblems and by applying the same MADM technique on subproblems

Now, we discuss the validity of our proposed MADM technique by testing the above criteria.

(1) Validity test by criterion 1: if we replace the decision values of a nonoptimal alternative $\mathscr{K}_{2}$ by $\widetilde{\mathscr{K}}_{2}$, then the new DM is given in Table 3.

By applying the GFFEWA operator for $\lambda=1$ and score function, the score values of alternatives are

$$
\begin{aligned}
& \mathcal{S}\left(\mathscr{B}_{1}\right)=0.15, \\
& \mathcal{S}\left(\widetilde{\mathscr{B}}_{2}\right)=-0.03, \mathcal{S}\left(\mathscr{B}_{3}\right)=0.34, \mathcal{S}\left(\mathscr{B}_{4}\right)=0.42 .
\end{aligned}
$$

The ranking of sanitizers is $\mathscr{K}_{4}>\mathscr{K}_{3}>\mathscr{K}_{1}>\widetilde{\mathscr{K}}_{2}$, which is the same as the original ranking order, and the best sanitizer is $\mathscr{K}_{4}$. Thus, our presented MADM model fulfills the test criterion 1.

(2) Validity test by criteria 2 and 3: for the validity of proposed algorithm, using criteria 2 and 3 , we split the problem into the smaller subproblems $\left\{\mathscr{K}_{1}, \mathscr{K}_{2}, \mathscr{K}_{4}\right\},\left\{\mathscr{K}_{1}, \mathscr{K}_{3}, \mathscr{K}_{4}\right\}$, and $\left\{\mathscr{K}_{2}, \mathscr{K}_{3}, \mathscr{K}_{4}\right\}$.
TABLE 3: Reconstructed FFDM.

\begin{tabular}{lccc}
\hline$\widetilde{\mathscr{E}}$ & $\mathcal{F}_{1}$ & $\mathcal{F}_{2}$ & $\mathcal{F}_{3}$ \\
\hline $\mathscr{K}_{1}$ & $(0.6,0.7)$ & $(0.6,0.2)$ & $(0.8,0.4)$ \\
$\widetilde{\mathscr{K}}_{2}$ & $(0.3,0.4)$ & $(0.5,0.4)$ & $(0.2,0.8)$ \\
$\mathscr{K}_{3}$ & $(0.8,0.3)$ & $(0.5,0.3)$ & $(0.6,0.4)$ \\
$\mathscr{K}_{4}$ & $(0.7,0.5)$ & $(0.8,0.1)$ & $(0.9,0.6)$ \\
\hline
\end{tabular}

TABLE 4: Distance of alternatives from FFPIS and FFNIS.

\begin{tabular}{lc}
\hline$D\left(\mathscr{K}_{l}, \mathcal{S}^{+}\right)$ & $D\left(\mathscr{K}_{l}, \mathcal{S}^{-}\right)$ \\
\hline 0.30 & 0.08 \\
0.46 & 0.29 \\
0.18 & 0.21 \\
0.09 & 0.32 \\
\hline
\end{tabular}

By utilizing the proposed technique, the ranking orders of alternatives in these subproblems are $\mathscr{K}_{4}>\mathscr{K}_{1}>\mathscr{K}_{2}, \mathscr{K}_{4}>\mathscr{K}_{3}>\mathscr{K}_{1}$, and $\mathscr{K}_{4}>\mathscr{K}_{3}>\mathscr{K}_{2}$, respectively. The combined ranking of alternatives is $\mathscr{K}_{4}>\mathscr{K}_{3}>\mathscr{K}_{1}>\mathscr{K}_{2}$, which is the same as that of the original ranking. Hence, the proposed MADM technique is authentic and proficient under criteria 2 and 3 .

\section{Comparison Analysis}

Here, we discuss the comparison of proposed theory with the FF TOPSIS method [5]. The steps to find out the best alternative by the FF TOPSIS method are

(1) Table 2 represents the FF decision matrix in which each entry corresponds to an FFN.

(2) The FF positive ideal solution (FFPIS) $\mathcal{S}^{+}$and FF negative ideal solution (FFNIS) $\mathcal{S}^{-}$are

$$
\begin{aligned}
& \mathcal{S}^{+}=\{(0.8,0.3),(0.8,0.1),(0.9,0.6)\}, \\
& \mathcal{S}^{-}=\{(0.6,0.7),(0.5,0.3),(0.3,0.6)\} .
\end{aligned}
$$

(3) The distance between the alternative $\mathscr{K}_{l}$ and FFPIS $\mathcal{S}^{+}$together with the FFNIS $\mathcal{S}^{-}$are given in Table 4 .

(4) The revised closeness degree of each alternative is given as

$$
\begin{gathered}
\xi\left(\mathscr{K}_{1}\right)=-3.05, \\
\xi\left(\mathscr{K}_{2}\right)=-4.11 \\
\xi\left(\mathscr{K}_{3}\right)=-1.28 \\
\xi\left(\mathscr{K}_{4}\right)=0.10 .
\end{gathered}
$$

(5) We get the following ranking list by arranging the alternatives in the decreasing order with respect to $\xi\left(\mathscr{K}_{l}\right)$ :

$$
\mathscr{K}_{4}>\mathscr{K}_{3}>\mathscr{K}_{1}>\mathscr{K}_{2}
$$

(6) $\mathscr{K}_{4}$ is the best alternative. 


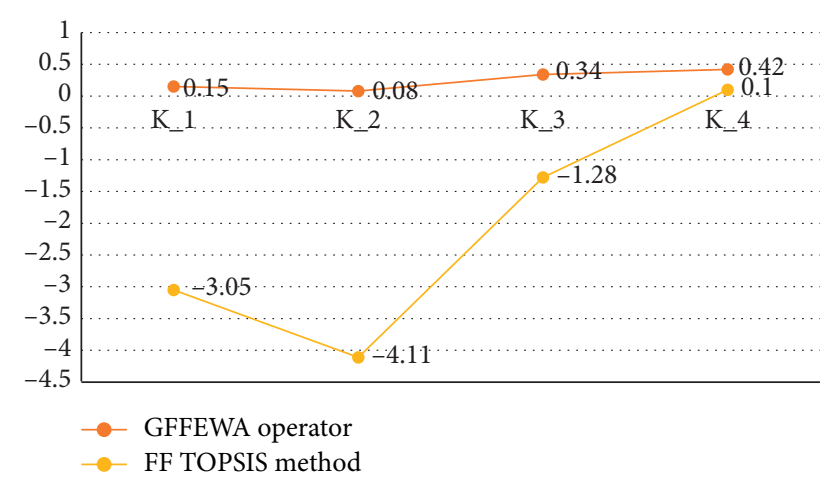

Figure 1: Comparison of the GFFEWA operator and FF TOPSIS method.
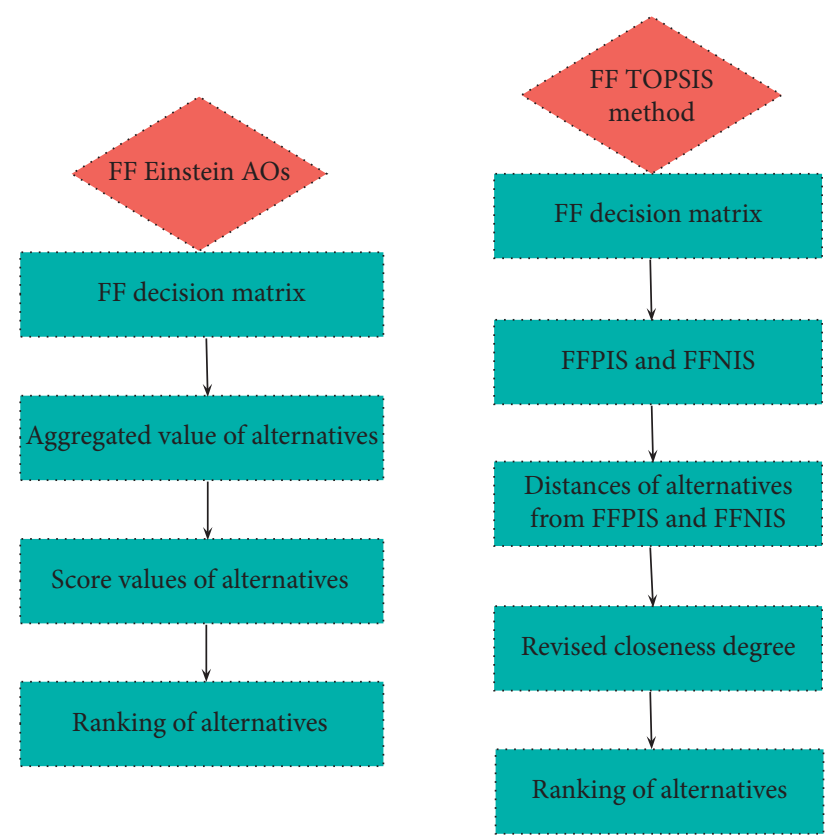

FIgURE 2: Flow chart for MADM problem using FF Einstein AOs and FF TOPSIS method.

From the outcomes of proposed operators and FF TOPSIS method, as shown in Figure 1, we conclude that ranking lists obtained from both compared methods are the same and the best alternative from both approaches is $\mathscr{K}_{4}$. The FF TOPSIS method is a good approach to solve DM problems but there are many hindrances which can be solved by using our proposed theory. The FF Einstein AOs are more flexible and easy approach. A best alternative can be obtained by a short process. The results from proposed theory are more accurate and closest to original results.

The steps to solve any MADM problem by FF Einstein AOs and FF TOPSIS method are shown in Figure 2.

10.1. Advantages and Limitations of Proposed Model. The proposed model is superior than the IF and PF models because it contains the space of IF and PF models. The cubic sum of membership and nonmembership degrees is bounded by 1 in the proposed model. The MADM approaches discussed in $[10,12,13,15]$ failed to handle the proposed application because $0.9+0.6>1$ and $0.9^{2}+0.6^{2}>1$ but proposed approach covers all such situations. The results are more precise and accurate by using the proposed model. However, there are some limitations of this model. It cannot be applied in the situations where we take the parameters for the evaluation of anything. It means this theory lacks parametrization property.

\section{Conclusions}

An FFS is an extension of IFS and PFS which has more flexible structure to solve decision-making problems owing to the condition $\mu^{3}+\nu^{3} \leq 1$. Moreover, Einstein's $t$-norm and $t$-conorm have more generalized structure that operates efficiently to integrate the intricate information. The limitations of existing operators and beneficial characteristics of Einstein AOs motivated us to endeavor for the development of a fruitful combination of Einstein AOs with FFNs.

A major contribution of the study is the development new tremendous AOs, called, FFEWA, FFEOWA, GFFEWA, and GFFEOWA operators. Some captivating properties of these operators have been discussed. Another achievement of this study is the establishment of a MADM technique on the basis of the proposed operators to manifest the application of the proposed operators. A MADM problem for the selection of an effective sanitizer to reduce the coronavirus has been presented to demonstrate the potency of the proposed strategy.

The validity test has been discussed to unfold the consistency of proposed work. A comparison analysis of our proposed theory with the FF TOPSIS method has been presented to exhibit the dominance of our proposed operators over the FF TOPSIS method. In short, this article builds up a tool that has the rich properties of Einstein AOs and flexibility of the FF model. In future, our aim is to develop some worthwhile AOs using the theoretical foundations of Einstein norms for the Fermatean fuzzy soft set.

\section{Data Availability}

No data were used to support this study.

\section{Conflicts of Interest}

The authors declare that they have no conflicts of interest regarding the publication of the research article.

\section{References}

[1] L. A. Zadeh, "Fuzzy sets," Information and Control, vol. 8, no. 3, pp. 338-353, 1965.

[2] K. T. Atanassov, "Intuitionistic fuzzy sets," Fuzzy Sets and Systems, vol. 20, no. 1, pp. 87-96, 1986.

[3] R. R. Yager, "Pythagorean fuzzy subsets," in Proceedings of the 2013 Joint IFSA World Congress and NAFIPS Annual Meeting (IFSA/NAFIPS), pp. 57-61, Edmonton, Canada, June 2013.

[4] R. R. Yager, "Generalized orthopair fuzzy sets," IEEE Transactions on Fuzzy Systems, vol. 25, no. 5, pp. 1222-1230, 2016. 
[5] T. Senapati and R. R. Yager, "Fermatean fuzzy sets," Journal of Ambient Intelligence and Humanized Computing, vol. 11, no. 2, pp. 663-674, 2020.

[6] Z. Xu, "Intuitionistic fuzzy aggregation operators," IEEE Transactions on Fuzzy Systems, vol. 15, no. 6, pp. 1179-1187, 2007.

[7] H. Zhao, Z. Xu, M. Ni, and S. Liu, "Generalized aggregation operators for intuitionistic fuzzy sets," International Journal of Intelligent Systems, vol. 25, no. 1, pp. 1-30, 2010.

[8] K. Rahman, A. Ali, M. Shakeel, M. S. A. Khan, and M. Ullah, "Pythagorean fuzzy weighted averaging aggregation operator and its application to decision-making theory," The Nucleus, vol. 54, no. 3, pp. 190-196, 2017.

[9] X. Zhao and G. Wei, "Some intuitionistic fuzzy Einstein hybrid aggregation operators and their application to multiple attribute decision making," Knowledge-Based Systems, vol. 37, pp. 472-479, 2013.

[10] W. Wang and X. Liu, "Intuitionistic fuzzy information aggregation using Einstein operations," IEEE Transactions on Fuzzy Systems, vol. 20, no. 5, pp. 923-938, 2012.

[11] X. Cai and L. Han, "Some induced Einstein aggregation operators based on the data mining with interval-valued intuitionistic fuzzy information and their application to multiple attribute decision making," Journal of Intelligent \& Fuzzy Systems, vol. 27, no. 1, pp. 331-338, 2014.

[12] H. Garg, "A new generalized Pythagorean fuzzy information aggregation using Einstein operations and its application to decision making," International Journal of Intelligent Systems, vol. 31, no. 9, pp. 886-920, 2016.

[13] H. Garg, "Generalized Pythagorean fuzzy geometric aggregation operators using Einsteint-norm andt-conorm for multicriteria decision-making process," International Journal of Intelligent Systems, vol. 32, no. 6, pp. 597-630, 2017.

[14] M. Akram, W. A. Dudek, and J. M. Dar, "Pythagorean Dombi fuzzy aggregation operators with application in multicriteria decision-making," International Journal of Intelligent Systems, vol. 34, no. 11, pp. 3000-3019, 2019.

[15] G. Shahzadi, M. Akram, and A. N. Al-Kenani, "Decisionmaking approach under Pythagorean fuzzy Yager weighted operators," Mathematics, vol. 8, no. 1, p. 70, 2020.

[16] P. Liu and P. Wang, "Some q-rung orthopair fuzzy aggregation operators and their applications to multiple-attribute decision making," International Journal of Intelligent Systems, vol. 33, no. 2, pp. 259-280, 2018.

[17] G. Wei, H. Gao, and Y. Wei, "Some q-rung orthopair fuzzy Heronian mean operators in multiple attribute decision making," International Journal of Intelligent Systems, vol. 33, no. 7, pp. 1426-1458, 2018.

[18] P. Liu, S.-M. Chen, and P. Wang, "Multiple-attribute group decision-making based on $q$-rung orthopair fuzzy power Maclaurin symmetric mean operators," IEEE Transactions on Systems, Man, and Cybernetics: Systems, vol. 50, no. 10, pp. 3741-3756, 2020.

[19] C. Jana, G. Muhiuddin, and M. Pal, "Some Dombi aggregation of $q$-rung orthopair fuzzy numbers in multiple-attribute decision making," International Journal of Intelligent Systems, vol. 34, no. 12, pp. 3220-3240, 2019.

[20] P. Liu and J. Liu, "Some q-rung orthopair fuzzy Bonferroni mean operators and their application to multi-attribute group decision-making," International Journal of Intelligent Systems, vol. 33, no. 2, pp. 315-347, 2018.

[21] B. P. Joshi and A. Gegov, "Confidence levels q-rung orthopair fuzzy aggregation operators and its applications to MCDM problems," International Journal of Intelligent Systems, vol. 35, no. 1, pp. 125-149, 2019.

[22] M. Akram and G. Shahzadi, "A hybrid decision-making model under $q$-rung orthopair fuzzy Yager aggregation operators," Granular Computing, pp. 1-15, 2020.

[23] P. Liu, M. Akram, and A. Sattar, "Extensions of prioritized weighted aggregation operators for decision-making under complex q-rung orthopair fuzzy information," Journal of Intelligent \& Fuzzy Systems, pp. 1-25, 2020.

[24] T. Senapati and R. R. Yager, "Some new operations over fermatean fuzzy numbers and application of fermatean fuzzy WPM in multiple criteria decision making," Informatica, vol. 30, no. 2, pp. 391-412, 2019.

[25] T. Senapati and R. R. Yager, "Fermatean fuzzy weighted averaging/geometric operators and its application in multicriteria decision-making methods," Engineering Applications of Artificial Intelligence, vol. 85, pp. 112-121, 2019.

[26] M. Akram, W. A. Dudek, and F. Ilyas, "Group decisionmaking based on pythagorean fuzzy TOPSIS method," International Journal of Intelligent Systems, vol. 34, no. 7, pp. 1455-1475, 2019.

[27] M. Akram, H. Garg, and K. Zahid, "Extensions of ELECTRE-I and TOPSIS methods for group decision-making under complex Pythagorean fuzzy environment," Iranian Journal of Fuzzy Systems, vol. 17, no. 5, pp. 147-164, 2020.

[28] M. Akram, N. Yaqoob, G. Ali, and W. Chammam, "Extensions of Dombi aggregation operators for decision-making under $m$-polar fuzzy information," Journal of Mathematics, vol. 2020, Article ID 4739567, 20 pages, 2020.

[29] M. Akram, F. Ilyas, and H. Garg, "Multi-criteria group decision making based on ELECTRE I method in Pythagorean fuzzy information," Soft Computing, vol. 24, no. 5, pp. 3425-3453, 2020.

[30] M. Akram, A. Bashir, and H. Garg, "Decision-making model under complex picture fuzzy Hamacher aggregation operators," Computational \& Applied Mathematics, vol. 39, p. 226, 2020.

[31] M. Akram, X. Peng, A. N. Al-Kenani, and A. Sattar, "Prioritized weighted aggregation operators under complex Pythagorean fuzzy information," Journal of Intelligent \& Fuzzy Systems, vol. 39, no. 3, pp. 4763-4783, 2020.

[32] M. Akram, G. Shahzadi, and X. Peng, "Extension of Einstein geometric operators to multi-attribute decision-making under q-rung orthopair fuzzy information," Granular Computing, 2020.

[33] J. C. R. Alcantud, "Characterization of the existence of maximal elements of acyclic relations," Economic Theory, vol. 19, no. 2, pp. 407-416, 2002.

[34] F. Feng, M. Liang, H. Fujita, R. Yager, and X. Liu, "Lexicographic orders of intuitionistic fuzzy values and their relationships," Mathematics, vol. 7, no. 2, p. 166, 20190.

[35] F. Feng, H. Fujita, M. I. Ali, R. R. Yager, and X. Liu, "Another view on generalized intuitionistic fuzzy soft sets and related multiattribute decision making methods," IEEE Transactions on Fuzzy Systems, vol. 27, no. 3, pp. 474-488, 2019.

[36] H. Garg, J. Gwak, T. Mahmood, and Z. Ali, "Power aggregation operators and VIKOR methods for complex q-rung orthopair fuzzy sets and their applications," Mathematics, vol. 8, no. 4, p. 538, 2020.

[37] H. Garg, G. Shahzadi, and M. Akram, "Decision-making analysis based on Fermatean fuzzy Yager aggregation operators with application in COVID-19 testing facility," Mathematical Problems in Engineering, vol. 2020, Article ID 7279027, 16 pages, 2020. 
[38] P. Liu, G. Shahzadi, and M. Akram, "Specific types of $q$-rung picture fuzzy Yager aggregation operators for decisionmaking," International Journal of Computational Intelligence Systems, vol. 13, no. 1, pp. 1072-1091, 2020.

[39] P. Liu, Q. Khan, and T. Mahmood, "Group decision making based on power Heronian aggregation operators under neutrosophic cubic environment," Soft Computing, vol. 24, no. 3, pp. 1971-1997, 2020.

[40] X. Ma, M. Akram, K. Zahid, and J. C. R. Alcantud, "Group decision-making framework using complex Pythagorean fuzzy information," Neural Computing and Applications, pp. 1-21, 2020.

[41] X. Peng, J. Dai, and H. Garg, "Exponential operation and aggregation operator for q-rung orthopair fuzzy set and their decision-making method with a new score function," International Journal of Intelligent Systems, vol. 33, no. 11, pp. 2255-2282, 2018.

[42] X. Peng and Y. Yang, "Fundamental properties of intervalvalued Pythagorean fuzzy aggregation operators," International Journal of Intelligent Systems, vol. 31, no. 5, pp. 444-487, 2016.

[43] D. Rani and H. Garg, "Complex intuitionistic fuzzy power aggregation operators and their applications in multi-criteria decision-making," Expert Systems, vol. 35, no. 6, Article ID e12325, 2018.

[44] K. Ullah, H. Garg, T. Mahmood, N. Jan, and Z. Ali, "Correlation coefficients for T-spherical fuzzy sets and their applications in clustering and multi-attribute decision making," Soft Computing, vol. 24, no. 3, pp. 1647-1659, 2020.

[45] N. Waseem, M. Akram, and J. C. R. Alcantud, "Multi-attribute decision-making based on m-polar fuzzy Hamacher aggregation operators," Symmetry, vol. 11, no. 12, p. 1498, 2019.

[46] G. Wei and M. Lu, "Pythagorean fuzzy Maclaurin symmetric mean operators in multiple attribute decision making," International Journal of Intelligent Systems, vol. 33, no. 5, pp. 1043-1070, 2018.

[47] G. Wei and M. Lu, "Pythagorean fuzzy power aggregation operators in multiple attribute decision making," International Journal of Intelligent Systems, vol. 33, no. 1, pp. 169-186, 2018.

[48] R. R. Yager, "Aggregation operators and fuzzy systems modeling," Fuzzy Sets and Systems, vol. 67, no. 2, pp. 129-145, 1994.

[49] R. R. Yager, "Pythagorean membership grades in multi-criteria decision-making," IEEE Transactions on Fuzzy Systems, vol. 22, no. 4, pp. 958-965, 2013.

[50] Z. Yang, X. Li, H. Garg, and M. Qi, "Decision support algorithm for selecting an antivirus mask over COVID-19 pandemic under spherical normal fuzzy environment," International Journal of Environmental Research and Public Health, vol. 17, no. 10, p. 3407, 2020.

[51] S. Zeng, J. Chen, and X. Li, “A hybrid method for Pythagorean fuzzy multiple-criteria decision making," International Journal of Information Technology \& Decision Making, vol. 15, no. 2, pp. 403-422, 2016.

[52] J. Zhan, B. Sun, and X. Zhang, "PF-TOPSIS method based on CPFRS models: an application to unconventional emergency events," Computers \& Industrial Engineering, vol. 139, Article ID 106192, 2020.

[53] X. Wang and E. Triantaphyllou, "Ranking irregularities when evaluating alternatives by using some ELECTRE methods," Omega, vol. 36, no. 1, pp. 45-63, 2008.
[54] K. Bai, X. Zhu, J. Wang, and R. Zhang, "Some partitioned Maclaurin symmetric mean based on q-rung orthopair fuzzy information for dealing with multi-attribute group decision making," Symmetry, vol. 10, no. 9, p. 383, 2018.

[55] A. Fahmi, F. Amin, S. Abdullah, and A. Ali, "Cubic fuzzy Einstein aggregation operators and its application to decisionmaking," International Journal of Systems Science, vol. 49, no. 11, pp. 2385-2397, 2018.

[56] A. Khan, S. Ashraf, S. Abdullah, M. Qiyas, J. Luo, and S. Khan, "Pythagorean fuzzy Dombi aggregation operators and their application in decision support system," Symmetry, vol. 11, no. 3, p. 383, 2019.

[57] X. Peng and Y. Yang, "Some results for Pythagorean fuzzy sets," International Journal of Intelligent Systems, vol. 30, no. 11, pp. 1133-1160, 2015.

[58] X. Peng and H. Yuan, "Fundamental properties of Pythagorean fuzzy aggregation operators," Fundamenta Informaticae, vol. 147, no. 4, pp. 415-446, 2016.

[59] X. Peng and G. Selvachandran, "Pythagorean fuzzy set: state of the art and future directions," Artificial Intelligence Review, vol. 52, no. 3, pp. 1873-1927, 2019. 